\title{
Article \\ The Italian Guidelines on Risk Classification and Management of Bridges: Applications and Remarks on Large Scale Risk Assessments
}

\author{
Giuseppe Santarsiero*(-), Angelo Masi, Valentina Picciano $(\mathbb{D})$ and Andrea Digrisolo (1) \\ School of Engineering, University of Basilicata viale dell'Ateneo Lucano, 10, 85100 Potenza, Italy; \\ angelo.masi@unibas.it (A.M.); valepicc95@gmail.com (V.P.); andrea.digrisolo@unibas.it (A.D.) \\ * Correspondence: giuseppe.santarsiero@unibas.it
}

Citation: Santarsiero, G.; Masi, A.; Picciano, V.; Digrisolo, A. The Italian Guidelines on Risk Classification and Management of Bridges: Applications and Remarks on Large Scale Risk Assessments. Infrastructures 2021, 6, 111. https://doi.org/10.3390/ infrastructures6080111

Academic Editor: Marinella Fossetti

Received: 1 July 2021

Accepted: 4 August 2021

Published: 9 August 2021

Publisher's Note: MDPI stays neutral with regard to jurisdictional claims in published maps and institutional affiliations.

Copyright: (c) 2021 by the authors. Licensee MDPI, Basel, Switzerland. This article is an open access article distributed under the terms and conditions of the Creative Commons Attribution (CC BY) license (https:// creativecommons.org/licenses/by/ $4.0 /)$.
Abstract: Bridges are essential for guaranteeing the functioning of transportation systems since their failure can cause serious threats to the safety, well-being and economy of modern communities, especially in emergency conditions. Following recent bridge failures, among which include the Morandi bridge in 2018, specific guidelines on risk classification and management, safety assessment and monitoring of existing bridges have been issued in Italy by the Minister of Infrastructure as a mandatory code. They pay particular attention to the evaluation of the residual life span of critical transportation infrastructure dating back to the 1950s and 1960s of the last century. Being a newly issued tool, the Guidelines need to be applied and tested in order to find possible drawbacks and to point out the main factors influencing their results. Therefore, in this study, after a short description of the Italian Guidelines, pointing out some differences with other approaches adopted worldwide, some advantages and disadvantages are discussed by an application to a bridge stock located in the Basilicata region (Italy). Data needed to apply the Guidelines are gathered by a purposely set up procedure that exploits existing databases on road infrastructure (OpenStreetMap) complemented by additional data retrieved by means of public image repositories (Google Street View). By accounting for the qualitative nature of the risk classification results obtained by applying the lower analysis levels of the Guidelines, a prioritization method is proposed for ranking bridges for higher assessment levels and possible interventions, as well as consequent funds allocation. The application shows that the Guidelines' approach tends to provide conservative results. In fact, even in case of bridges with low degradation levels, the final risk classification induces actions undertaken for preliminary or detailed assessment; thus, normal operation (with periodic inspections) would not be possible anymore.

Keywords: bridges; risk classification; risk management; prioritization; safety assessment; inspections

\section{Introduction}

The serviceability and safety of critical transportation infrastructures such as bridges are of paramount importance for modern communities. When people travel for business or for pleasure, they take the safety of the road infrastructure for granted even though many bridges, tunnels and ancillary structures have already exceeded their service life [1] In fact, aggressive environmental conditions and increasing traffic volume can cause significant deterioration to many bridges. Timely maintenance measures are crucial in order to ensure a satisfactory performance of transportation networks over their life span, taking into account that due to high construction costs, limited redundancy of major roadways frequently exists, especially in mountain areas. When a bridge is unpassable due to excessive deterioration or damage caused by natural events and detours are rendered necessary, road alternatives do not always offer the same level of effectiveness in terms of time and cost of transportation. Therefore, in order to maintain an adequate level of efficiency in road infrastructure, frequent maintenance interventions are required to avoid 
invasive rehabilitation works, inducing high impacts on traffic management and safety. This is even clearer after the Morandi bridge collapse that killed 43 people in August 2018 [2,3] and forced motorway traffic to take an urban detour with consequent jams. Therefore, aging and degradation effects must be seriously considered in order to evaluate the residual life span of each bridge structure [4].

Italian bridges are owned and managed by various (local and national) bodies that use in-house management systems with different levels of complexity. Their bridge management systems (BMS) are devoted, first of all, to managing and updating the bridge inventory, to recording data upon inspections, to planning short-term and long-term interventions and finally to optimising global fund allocation by considering the available information on the stock [5]. As can be observed in Section 2, currently available decisionmaking processes based on BMSs are unfortunately only about internal evaluations, i.e., based on the bridge condition and rarely considering external factors such as flooding and landslide hazards [6]. This means that a more holistic approach is necessary towards the consideration of all the risks that can affect bridges and, moreover, a uniform approach should be used at the national level in order to allow the interoperability of databases owned by different management bodies. This could result in a unified approach for risk evaluation and management of bridges over the country, which would be helpful in prioritizing interventions in a rational manner (e.g., [7-10]).

From this perspective, the current Italian technical code provisions [11] regarding existing structures are mainly focused on buildings, while the commentary [12] provides some rules for the safety assessment of existing bridges under seismic actions without indications about the assessment under service loads. In addition, current Eurocodes provide specific rules for newly designed bridges [13], while EC8-3 [14], which is specifically devoted to the assessment and retrofitting of existing structures, deals only with buildings, although the revision in progress in 2021 also includes bridge structures.

Therefore, in order to standardize the evaluation protocols devoted to existing bridges for both regional scale and individual bridge assessment, the Superior Council of Public Works (as part of the Ministry of Infrastructure) issued the Guidelines on risk classification and management, safety assessment and the monitoring of existing bridges [15]. Moreover, the Guidelines were approved as a technical code through the Ministry of Infrastructure Decree n. 578 (17 December 2020) [16].

The Guidelines' approach faces the problem of existing bridges through a multilevel procedure proceeding from risk evaluations at regional scale (based on qualitative risk assessment of bridges) to defining rules for detailed assessment procedures. More importantly, it is an approach that accounts for various types of risk deriving from structural vulnerabilities and from the surrounding environment. Specifically, the considered risks are those related to the following: structure-foundation, earthquakes, landslide and flood. Therefore, it is a multi-risk approach.

In this paper, the innovative multi-risk and multi-level approaches of the Italian Guidelines are shown after a description of the bridge risk evaluation methods and then applied to a bridge stock within the Basilicata region located in southern Italy. After a brief review of methods to measure bridge health, the proposed methodology is explained in detail and then the bridge stock analysed is then identified, and its characteristics are described. The aim is to find possible drawbacks related to an extensive use of this risk evaluation approach, which could be detrimental to an effective management of the wide Italian bridge stock.

The bridge inventory to which the Guidelines apply to is built through a purposely developed semi-automatic procedure able to collect data related to the bridge stock under consideration by exploiting existing databases on road infrastructure, such as OpenStreetMap (OSM) [17], complemented by further data retrieved by means of publicly available image repositories (Google Maps). Further development and implementation of such procedure at the national level could help in building a homogenous bridge inventory, which could also be openly accessible to road users for gathering information on road passability, as 
already performed in the USA with the National Bridge Inventory (NBI), which is an advanced database with a standard format [18].

This procedure is part of the innovative methodologies that allow the acquisition of data related to the damage conditions [19-24] of individual bridges but, more generally, of all the information that can be retrieved from images of urban scenes (structural type, material, static scheme, etc.) with the advantage of rapid acquisition and overcoming the disadvantages related to classical methods such as visual inspection or continuous monitoring $[25,26]$.

Once the necessary data have been collected, 48 bridges mostly built in the early 1970s are subjected to evaluation according to the Guidelines and the results obtained are analysed and discussed in order to identify any criticalities.

\section{Review of Methodologies for Bridge Health Assessment}

Several methods exist for the assessment of the health condition of a bridge and for the prioritization of maintenance and/or rehabilitation of a bridge stock within a given network. Some methods are based on risk assessment (risk-based approach) [1,6,27-29] as a product of hazard, vulnerability and exposure. In most cases, however, only the risk towards structural deficiency and/or seismic events is considered. Nevertheless, given the importance of infrastructure networks from a socio-economic, strategic and emergency points of view, the need to use multi-risk methods based on a holistic approach that takes into account any issues that may arise is stressed.

In other cases, methods based on risk assessment merely categorises bridges on a typological basis, i.e., it is considered, for example, that bridges belonging to the same structural type or having the same material may have the same behaviour or performance in the case of an earthquake. This is the case of the well-known HAZUS method [27] that is used for seismic risk assessment through the construction of fragility curves.

In this respect, therefore, a holistic approach must not only consider all possible risks (structural, seismic, flooding and landslide) but must also assess the health of bridges starting from the identification of their level of degradation. In this context the methods based on the calculation of an overall index, starting from the results of element-level inspections and taking into account other aspects, provide the level of health of the bridge at hand. Bridge Health Index or Bridge Condition Index [30], Bridge Overall Priority Index [31], Priority Index [32], Defect-based Urgency Index [33] and Integrated Bridge Index [29] represent examples of this type of approach used to assess the health or condition of the single structure, both in quantitative and qualitative terms, and to classify the bridges of the same network and, thus, prioritize interventions and the allocation of available funds.

The various existing indices differ from one another mainly in terms of the type of information and the amount of data considered for the index calculation and in the different computational approaches used. Four different approaches can be distinguished and are discussed below [6]:

- Ratio-based condition approaches: Those based on a numerical index given essentially by the ratio between a value representing the current condition of a bridge and one representing its condition just after its construction assumed as the reference baseline (i.e., 100\% performance). This type of index is frequently used in the United States, Canada, Italy and Japan [30]. This approach returns the residual economic value of a bridge and somewhat provides information on how much higher the current risk level is compared to a new structure, helping to undertake the required measures (loads reduction and decommissioning). One of the advantages is that bridges are analysed at the level of each structural component by identifying and assessing its deterioration. The resulting numerical index is very useful for comparing the health status of bridges belonging to the same network and, thus, prioritize interventions.

- Weighted average approaches: In a similar manner to the previous method, an overall numerical index indicating the level of health of the bridge is evaluated. The main difference with the previous method concerns the value assigned to the single structural 
element for the calculation of the index. In fact, in the previous case, those elements for which a possible failure implies a higher cost at the intervention level (failure cost) [6] are taken into account, while in this approach each structural element is evaluated according to its contribution to the global integrity of the structure by assigning an importance factor to each one (this approach is used in most countries: the United Kingdom, South Africa, Australia, Austria and Finland). The final numerical index, therefore, is the result of a weighted average of the conditions of the single structural element investigated.

- Worst-conditioned component approaches: In this approach, only the structural elements that represent the weakest point of the bridge or the most critical parts are considered for the calculation of the overall numerical index. This type of approach is very useful for assessing the vulnerability of a structure in case of a disaster or for identifying high-risk bridges because the health status of the whole bridge is related to that of its weakest parts. The main disadvantage is that it is not possible to have an overall picture of bridge deterioration by monitoring and recording only the state of the most critical elements (Germany and Japan [34]).

- Qualitative methods: These methods are no longer based on the calculation of a numerical index, but on a qualitative, direct and descriptive assessment of the state of health of a bridge [6]. This type of approach is used, therefore, to mainly identify the structures in need of maintenance and not for prioritization, since it does not provide a numerical scale necessary for the classification of bridges in the same network (Australia and Austria).

Other existing indices or methods do not belong to one of these categories but are the result of a combination of the four approaches described [29,31,32].

Damage-assessment-based methods, as already underlined, have the advantage of providing an overall picture of the deteriorated condition of an individual bridge, but they carry an important component related to the subjectivity and uncertainty of the inspection results at the level of the individual element. As these results strongly rely on human judgement, one inspector is likely to assess the condition of one element differently from another inspector [6]. It is important, therefore, to invest in scoring methods that provide results that are as objective as possible or that take the associated uncertainties into account. A solution to this problem could be the use of automatic damage detection methods by using non-destructive techniques or continuous monitoring sensors [35-40] or the use of Deep-Learning-Based Classification Techniques [19,20] and Computer VisionBased Inspection and Monitoring [25]. Deep-Learning-Based Classification Techniques are advanced methods based on the use of artificial intelligence for the recognition of information related to the degradation conditions of bridges. Computer Vision-Based Inspection and Monitoring techniques are based on the acquisition of images and/or videos by using remote cameras and unmanned aerial vehicles (UAVs) and data processing through algorithms that allow the images or video data to be automatically converted into usable information (presence, type, extent of degradation, etc.).

Moreover, the calculation of these numerical indices in most cases often consider only aspects related to structural damage, without considering other aspects such as the functionality or strategic nature of the structure (exposure) and, most importantly, does not allow the holistic evaluation of bridge risk since no information is considered regarding flooding or landslide risks [1].

In order to cover these shortcomings, the Italian Guidelines combine the advantages of damage-assessment-based methods at the element-level with the advantages of risk-based methods according to a more holistic approach. In particular, the prioritization strategy within the Guidelines is not linked to the calculation of a numerical index representative of the bridge health conditions. Similarly to qualitative methods mentioned before, a class of attention is assigned to each bridge, which can be high, medium-high, medium, medium-low or low. The bridges, which require urgent intervention or detailed assessment or rehabilitation, are chosen according to the resulting class of attention, without being able 
to construct a ranking for interventions since several bridges belonging to the same network may fall within the same class of attention. In addition, the level of degradation of the structure is considered in the assessment of structure-foundation and seismic vulnerability. Starting from the damage found on individual structural elements of the bridge, the approach proposed by the Guidelines evaluates the level of degradation of the entire bridge relating to the worst defects or damages found and whether they are on a key component for structural safety (e.g., prestressing tendons and dapped ends of girders). Therefore, the method falls within the element-level inspection methods such as BHI or $\mathrm{BCI}$ and, in particular, relating to the worst-conditioned component approaches, which have been described above. In fact, a high vulnerability is assigned to the bridge when defects are found on critical elements for the structural and seismic risks, whatever the extent of the degradation.

Stressing the importance of prioritizing bridges belonging to the same network to provide decision-making bodies with the basis for the allocation of available funds for repair and/or maintenance interventions, it is worth noting that the qualitative method provided by the Guidelines based on only five possible results for the bridge class of attention needs to be complemented by the consideration of additional aspects. In fact, functional efficiency and client impact factor (level of criticality in terms of socio-economic, political and historical considerations) [32] can further guide the decision-making process. For example, there are specifically designed methods able to simplify and automate this type of management that are based on multiple criteria evaluation: the Analytic Hierarchy Process (AHP) [9,41-43] is a method that has attracted the interest of many managers of infrastructure networks who have to take responsibility for such decisions. The method is based on the construction of a matrix for which its rows and columns represent the decision alternatives and the different evaluation criteria; by using particular mathematical axioms, the priority decision alternative is established through the evaluation of the weight of each chosen evaluation criterion.

In this study, a simple prioritization method is proposed and applied to the bridge stock under examination after the class of attention has been assigned to each bridge. It only considers two parameters that are not properly exploited in the class of attention's evaluation of individual bridges: the bridge's total length and the duality (i.e., the existence of two separate bridge structures for each carriageway).

\section{Dataset}

\subsection{The Italian Bridge Stock}

There are many road management bodies over the country. The most important ones are ANAS (Autonomous National Company of Road Management) and AISCAT (the association of private motorway concessionaries companies). ANAS manages a road network more than 29,000 km long. Most of this network is made up of highways (about $22,600 \mathrm{~km}$ ) and motorways (1300 km) [44]. About 13,000 bridges are managed by ANAS. The AISCAT associated companies manage about $5200 \mathrm{~km}$ of motorways with an unknown number of bridges. Other road management bodies are the 107 Italian territorial provinces and more than 8000 municipalities which are responsible for many local roads.

Thus, many stakeholders are involved in the management of Italian bridges whose number is approximately equal to more than 120,000 (Figure 1). Considering that the Guidelines are applicable only to bridges with a span length larger than $6.0 \mathrm{~m}$, the actual number of bridges could be lower than 120,000. Furthermore, it is also known that about 1400 bridges have no owners, meaning that no one oversees their surveillance and maintenance. This may happen in the case of a change in ownership between two road management bodies, which is not completed due to bureaucratic issues. 


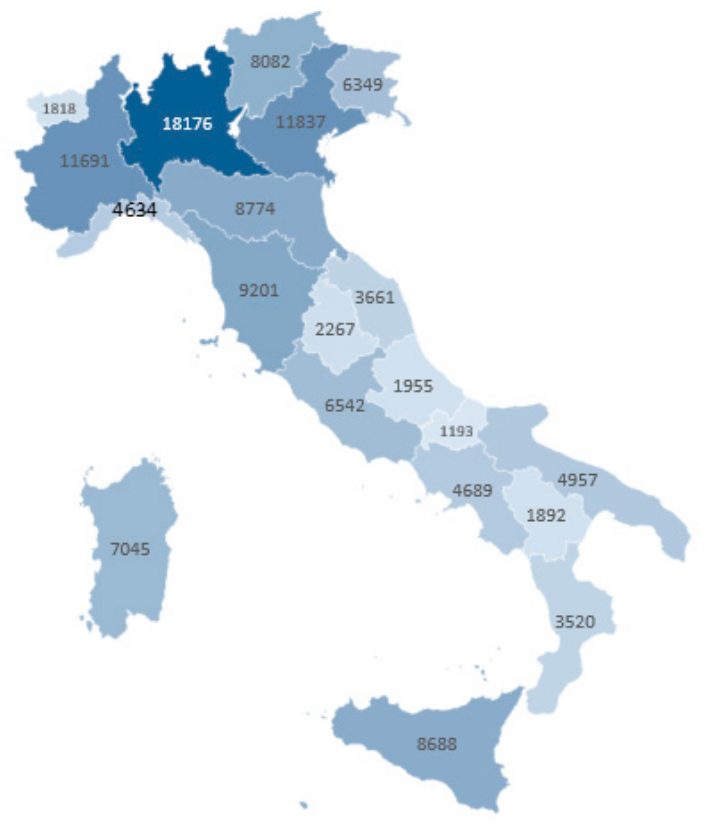

(a)

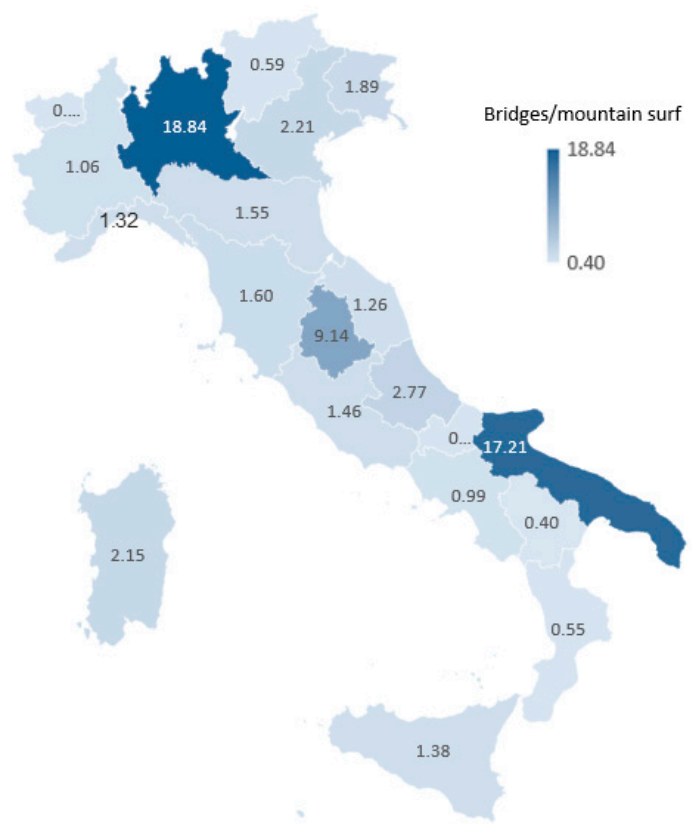

(b)

Figure 1. Distribution of Italian road bridges: (a) absolute number and (b) number of bridges per square kilometre of mountain surface.

The Italian Ministry of Infrastructure set up the AINOP web portal [45], which is a national database concerning public structures and infrastructures. The website is currently only accessible to infrastructure owners or management agencies in order to provide data helping to build the national archive. In the future, this database will be partly available to the public as open data.

As a consequence of the above-mentioned fragmented road ownership, different protocols are used to manage bridge stocks, carry out periodic surveys and to collect information. Moreover, storage and analysis procedures of bridge degradation data are also based on different BMSs, which can result in inhomogeneous decisions across the country with poor interoperability between different agencies. The enforcement of the Guidelines should be able to provide a unified approach in this direction.

The grade of infrastructure across the country has a high variability. As an example, out of about 120,000 Italian road bridges, more than 18,000 are in Lombardy region (9.97 million inhabitants), which is highly industrialized with a per capita GDP of about EUR 39 k. Only 1890 bridges are present in Basilicata region (548.000 inhabitants), which is much less industrialized with an almost half per capita GDP compared to Lombardy [46] (see Figure 1a). The number of bridges per 100,000 inhabitants is equal to 180 and 340 for the Lombardy and Basilicata regions, respectively. This could result in the conclusion that Basilicata has a higher grade of infrastructure. However, computing the ratio between the number of bridges and the mountain surface (areas where bridges are most needed) of each region according to [47] (Figure 1b) yields that Lombardy has 18.84 bridges per mountain square kilometre, while Basilicata only has 0.40 , being the lowest value among all Italian regions.

In regions with such a low grade of infrastructure, the failure of a road bridge could lead to catastrophic consequences on the road traffic (especially in an emergency), due to the lack of suitable detours. Moreover, long term effects on people's well-being are caused by the lack of infrastructure systems, such as depopulation and emigration [47].

For this reason, the effective evaluation of priorities in terms of assessment and refurbishment needs is a key issue for future actions on the Italian bridge stock with respect to risk management and socio-economic development strategies. 


\subsection{Description of the Bridge Stock under Evaluation}

The bridge stock under consideration belongs to the State Road SS 658 connecting the towns of Potenza and Melfi in the Basilicata region (Southern Italy) with its $48 \mathrm{~km}$ long path. Along with SS 407 (Trunk) and RA 5 (Motorway), which cuts through the region from east to west (Figure 2), SS 658 is a key highway proceeding from Potenza (the capital town of Basilicata region) towards the north through a hilly territory with 48 bridge structures. SS 658 has a single carriageway and most of its path was built in the early 1970s and is now approaching 50 years of age. Only a minor part of the road was built in the first half of the 80s. It is important to consider that the Basilicata region was classified as seismic only after the 1980 Irpinia-Basilicata earthquake, and therefore bridges designed and built in the 70s have no seismic provision.

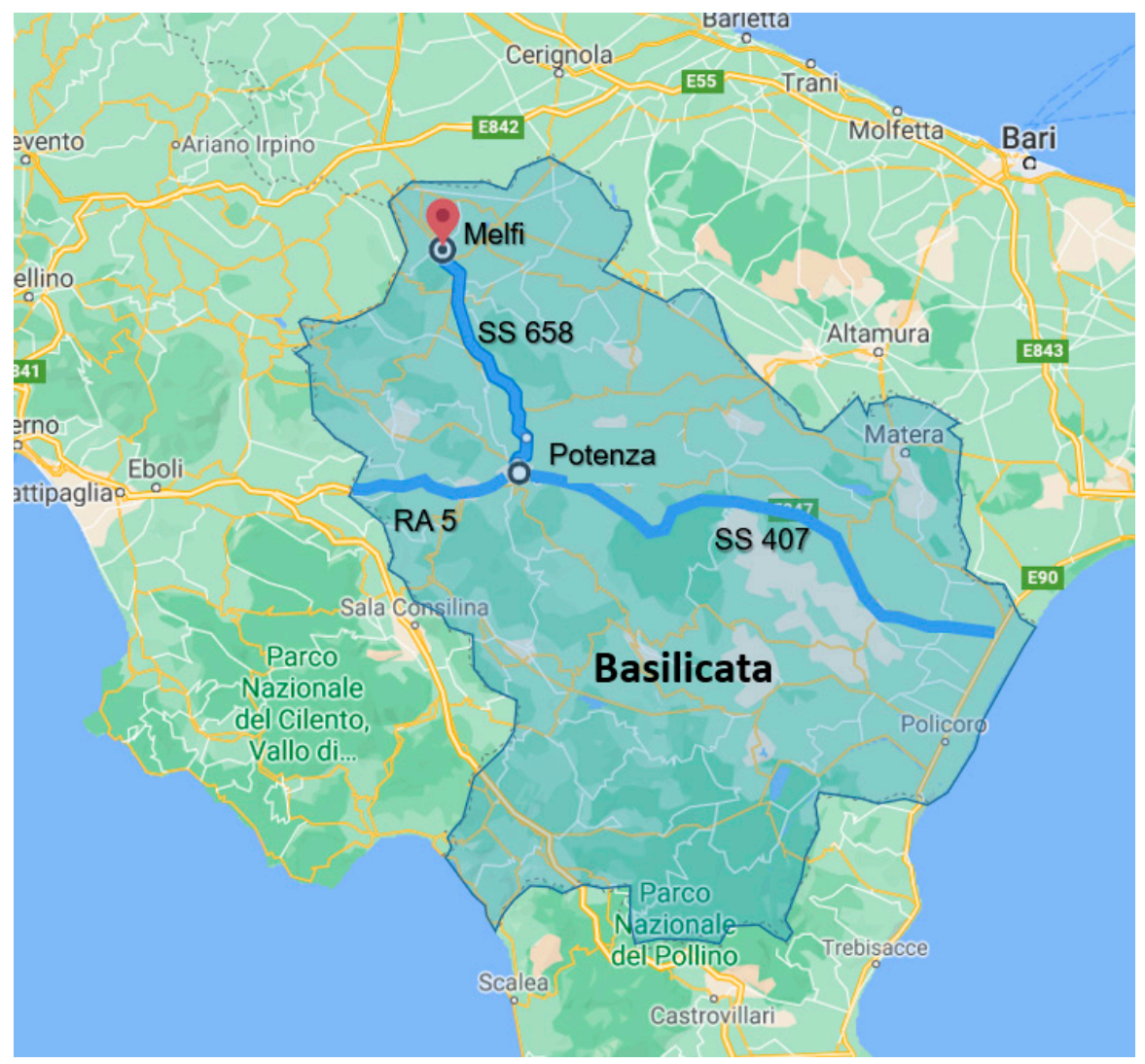

Figure 2. The SS 658 highway (map retrieved from Google Maps on March 2021).

Bridges constitute $28 \%$ of the total road route length, with their $13.5 \mathrm{~km}$ total development. This highlights the importance of proper risk classification and bridge stock management in order to retain the functionality and efficiency of this road.

As can be observed from Figure 3, most bridges are viaducts with a total length between 50 and $500 \mathrm{~m}$. Nine bridges have a total length smaller than $20 \mathrm{~m}$, being short overpasses on local or agricultural roads. Four bridges are more than $500 \mathrm{~m}$ long, and one viaduct is $4200 \mathrm{~m}$ long with its 118 spans. 


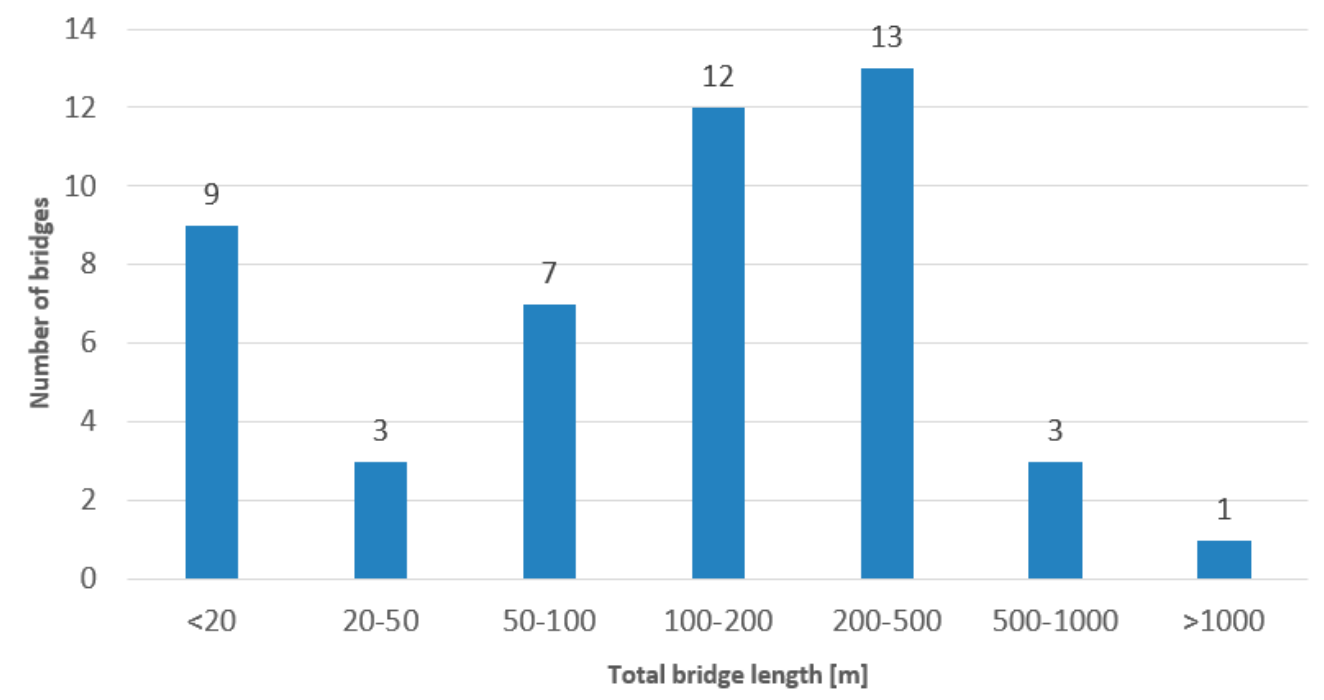

Figure 3. Distribution of bridge in total length classes.

The static scheme is mainly represented by the simply supported one (42 bridges). The continuous span is used only in one case (Figure 4).

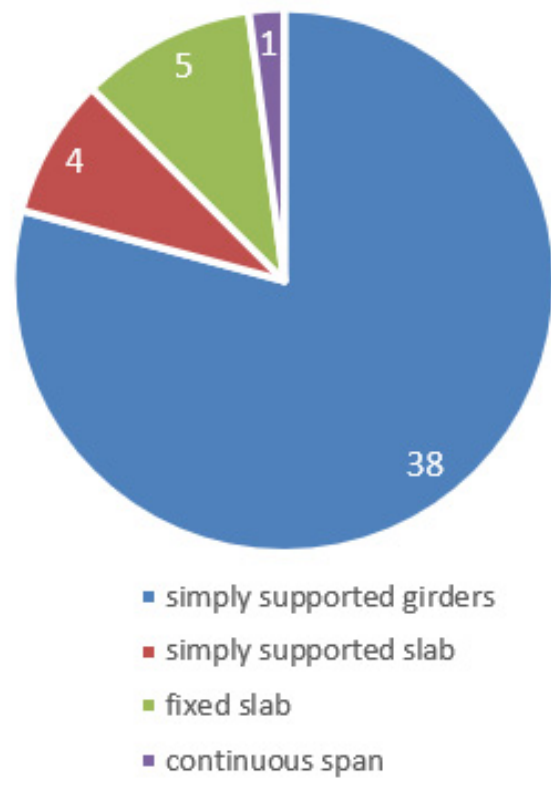

(a)

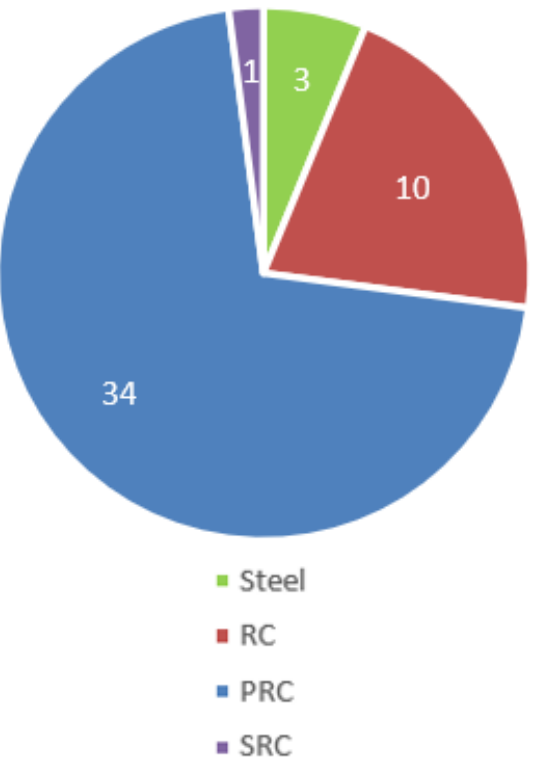

(b)

Figure 4. Distribution of bridges by static scheme (a) and deck material (b) $(R C=$ reinforced concrete; $P R C=$ prestressed reinforced concrete; SRC = steel reinforced concrete).

In most cases, the deck material comprises prestressed reinforced concrete (PRC). Reinforced concrete (RC) is used only for the small overpasses, while steel deck or mixed steel-RC (SRC) deck is used for the longest span bridges. Being a rather modern road, there are no masonry or iron bridges.

Table 1 shows the average value of the maximum span length found for each bridge deck material category. As expected, steel and SRC bridges are used for the longest bridges, even though they are usually part of viaducts with further approaching branches made of PRC spans. PRC bridges have a typical span length of about $34 \mathrm{~m}$. 
Table 1. Maximum span length as a function of deck material.

\begin{tabular}{cc}
\hline Deck Material & Average Maximum Span Length (m) \\
\hline Steel & 83 \\
RC & 6 \\
PRC & 34 \\
SRC & 60 \\
\hline
\end{tabular}

RC decks are used only for the smallest above-mentioned overpasses and have an average span length of $6 \mathrm{~m}$. Piers are always RC and have either hollow sections (Figure 5a) with the same deck's width or circular sections and cap beam (Figure 5c).
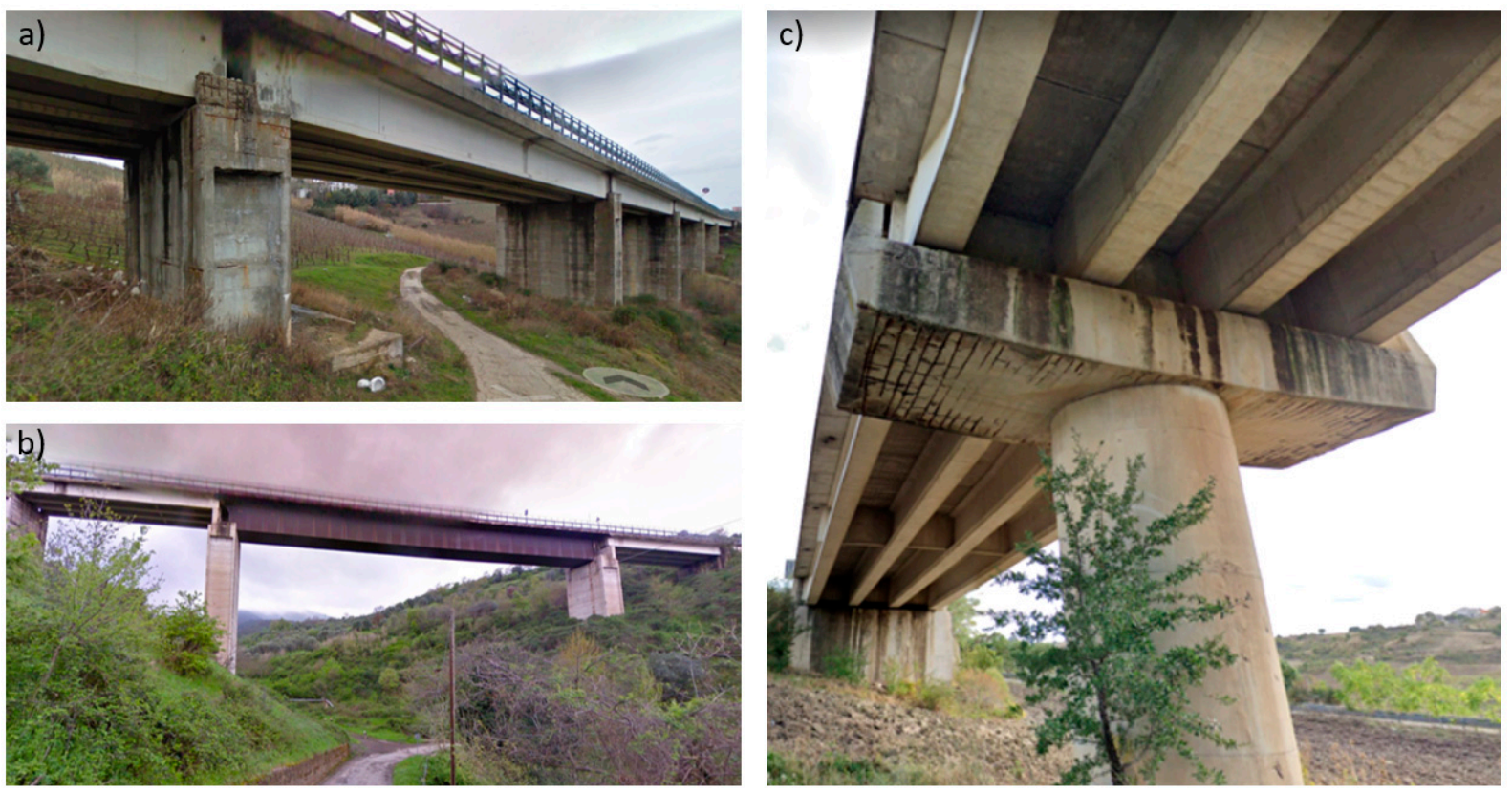

Figure 5. (a) Typical PRC viaduct, (b) steel span of a viaduct and (c) circular piers with cap beam.

\section{Methodology}

\subsection{Description}

First of all, the methodology proposed by the Italian Guidelines on risk classification and management, safety assessment and monitoring of existing bridges is summarized. The whole framework for risk classification and management is shown in Figure 6.

The methodology proposed by the Guidelines for ordinary bridge structures is based on five levels of analysis (from 0 to 4). As foreseen by the Guidelines, high importance bridges (i.e., important in terms of the socio-economic consequences of their collapse and for maintaining communications especially in emergency situations) should be analysed according to an additional level (5) and also by taking into account the network resilience. This study, being devoted to the assessment of the class of attention, will focus mainly on the first three levels ( 0 to 2 ) which are related to risk classification and management (Figure 6). Specifically, level 0 is devoted to gathering the available data on individual bridges (design documents and the results of periodic inspections), while level 1 foresees specific site inspections for verifying the current state of degradation and the possible presence of structural and non-structural components affected by significant defects. At level 2, exploiting the information collected through levels 0 and 1, each bridge is subjected to a procedure for the evaluation of the class of attention related to four different risk types: (i) structure-foundation, (ii) seismic, (iii) flooding and (iv) landslides. A qualitative risk evaluation is carried out, accounting for hazard, vulnerability and exposure. 


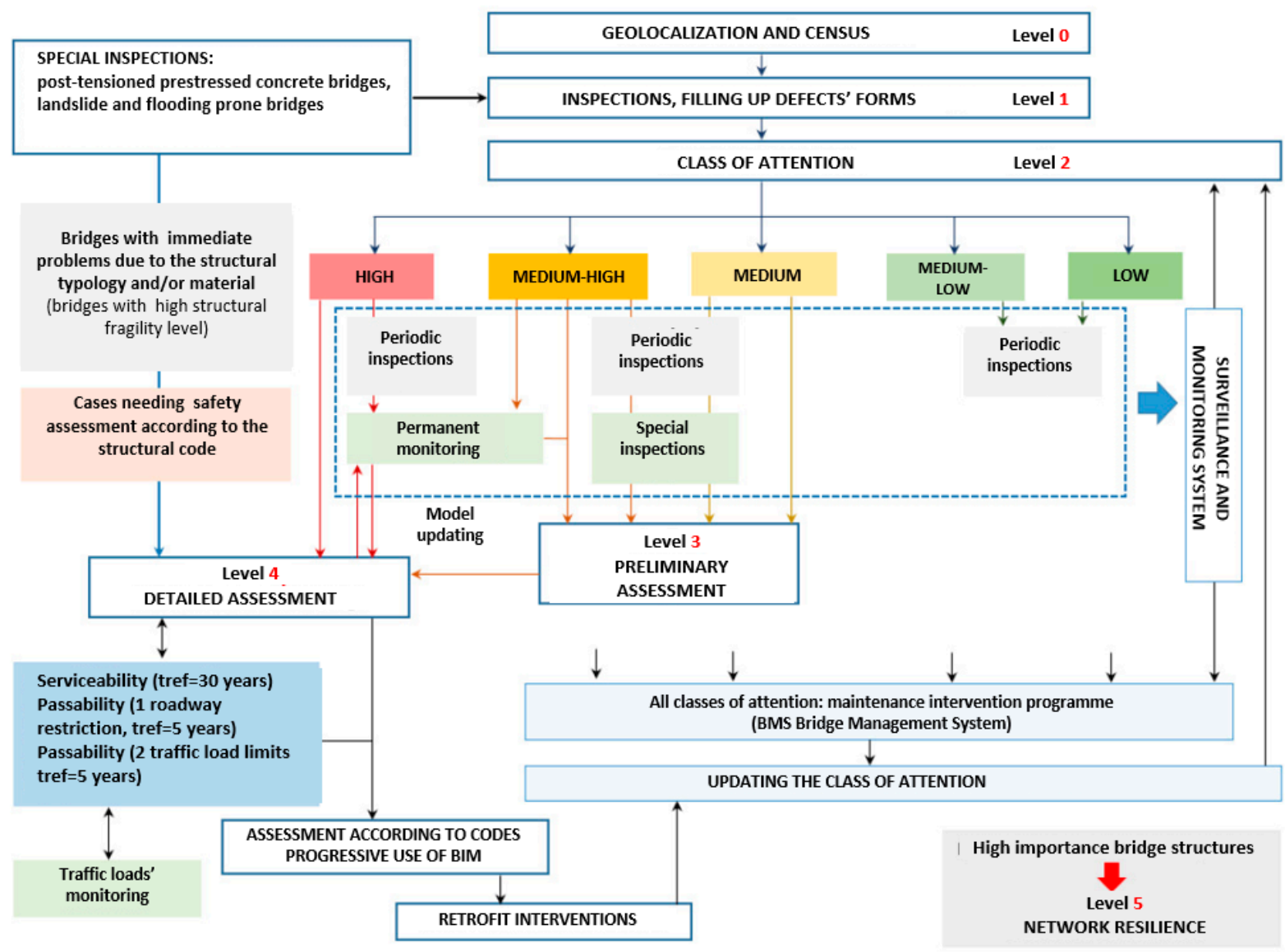

Figure 6. Multilevel approach and relationships among the different analysis levels [15].

Combining the results in terms of (partial) class of attention, an overall class of attention can be assigned to the bridge under examination according to five levels of increasing risk: low, medium-low, medium, medium-high and high. Based on this result, the bridge owner is required to adopt some consequent measures. As can be observed from Figure 6, in the case of a medium-low or low class of attention, the bridge can hold its serviceability by undergoing the usual periodic inspections. In other cases, more in-depth safety evaluations are required in order to make a decision related to either possible traffic load reduction measures or retrofit interventions.

It is worth noting that only structure-foundation and seismic risks will be explicitly considered in this study, since the above-mentioned databases cannot provide data related to landslide and flooding risks, for which reasonable assumptions will be made.

As for structure-foundation risks, hazard depends only on traffic loads (see Table 2). For the considered bridge stock (highway), the maximum loads prescribed by the current Italian structural code [11] will be considered without any reduction, because any type of vehicles must be allowed to pass these bridges. Therefore, hazard is always high for the bridge stock under examination. 
Table 2. Parameters influencing structure and foundations risk.

\begin{tabular}{ccc}
\hline $\begin{array}{c}\text { Risk } \\
\text { Component }\end{array}$ & $\begin{array}{c}\text { Primary } \\
\text { Parameters }\end{array}$ & $\begin{array}{c}\text { Secondary } \\
\text { Parameters }\end{array}$ \\
\hline Hazard & Extent of expected loads & - \\
\hline Vulnerability & $\begin{array}{c}\text { Level of degradation, static scheme (e.g., } \\
\text { arch and simply supported, dapped-end } \\
\text { beams), max span length, material (e.g., } \\
\text { RC, prestressed concrete, steel and } \\
\text { masonry) and number of spans }\end{array}$ & $\begin{array}{c}\text { Degradation speed } \\
\text { Design code }\end{array}$ \\
\hline Exposure & Average daily traffic and mean span length & $\begin{array}{c}\text { Road alternatives } \\
\text { Type of bypassed entity } \\
\text { Transport of dangerous goods }\end{array}$ \\
\hline
\end{tabular}

Vulnerability depends on the combination of the following parameters (see Figure 7):

- Level of degradation: to be evaluated by means of defect forms [15]. The level of degradation could sometimes be roughly evaluated by examining pictures retrieved from Street View when a road passes under the assessed bridge. This data is unreliable in most cases, and therefore the level of degradation will be assumed.

- Degradation speed: Degradation speed is evaluated by combining the level of degradation with the bridge's construction period. In fact, heavy degradation affecting a young bridge testifies a high degradation speed.

- Design code: It provides information of the design loads that are influencing the structural capacity of the bridge. A class of design codes usually includes more than one code.

- Combination of static scheme, maximum span length and material: It provides information on the structure and foundation vulnerability that is determined based on a specific combination table reported in the Guidelines.

Finally, exposure is basically evaluated by the number of vehicles passing over the bridge daily. This information has been retrieved through the ANAS (bridge stock owner) web page, referring to the average daily traffic data for the year 2018 [48].

The possible road alternatives are considered absent since that would require a specific transportation study. The bypassed entity (e.g., river, railway and road) is also considered as a parameter affecting the consequences of an eventual bridge collapse and, therefore, the exposure. The possibility of transport of dangerous goods is not considered in this study given the absence of data. 


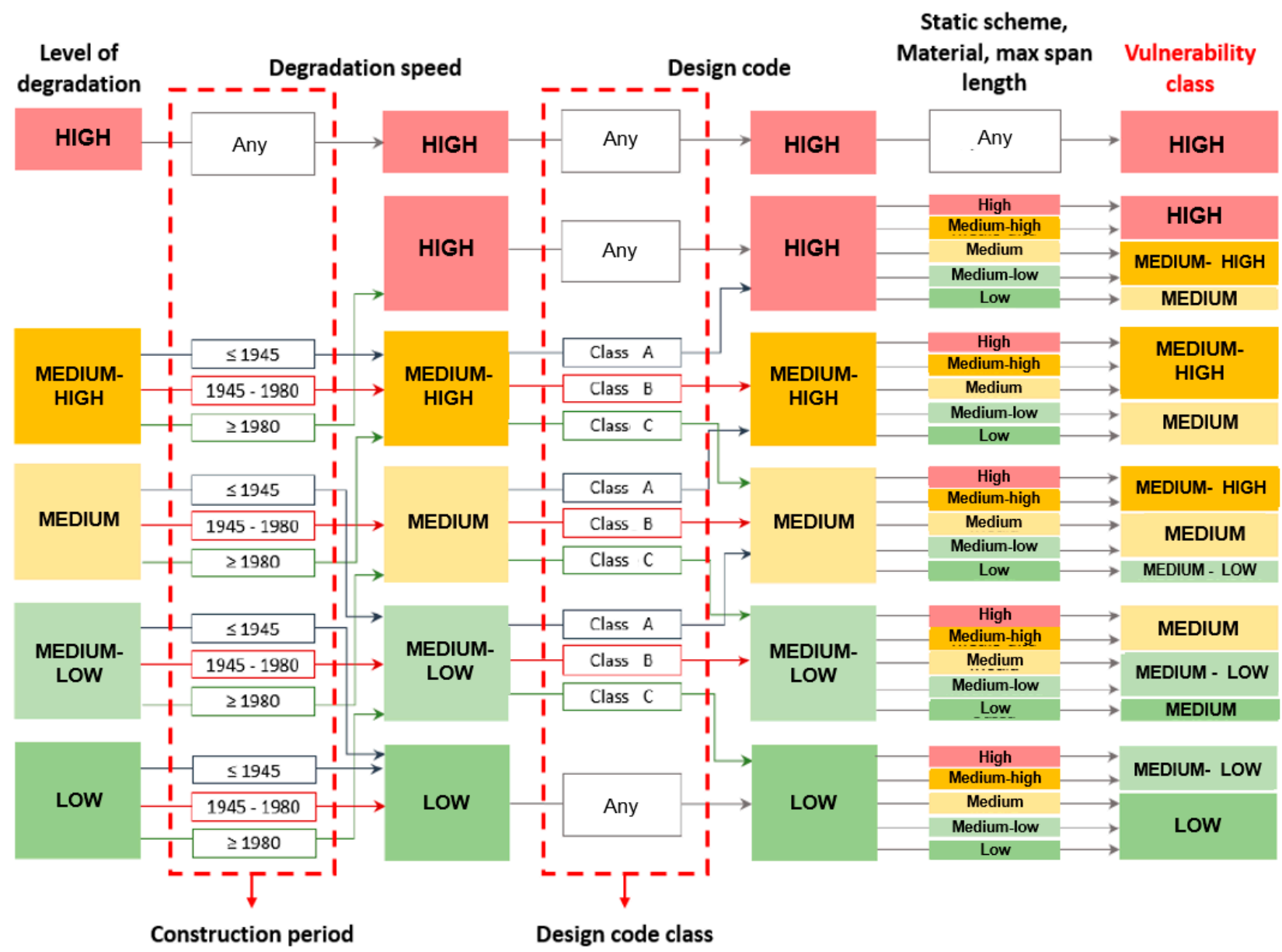

Figure 7. Logical path for the determination of the vulnerability class [15].

Once geographic identification of the bridge is made by using OMS data (parameters 1 and 2 in Table 3), other data can be retrieved through a remote survey by using Google Maps and the related Street View function.

Table 3. Data for vulnerability and exposure evaluations.

\begin{tabular}{ccc}
\hline ID & Parameter & Source \\
\hline 1 & Geographic coordinates & OpenStreetMap \\
2 & Road's ID code & OpenStreetMap \\
3 & Road's year of construction & Italian highway road archive \\
4 & Load values & Current code \\
5 & Level of degradation & Assumption \\
6 & Static scheme & Google Maps \\
7 & Max span length & Google Maps \\
8 & Number of spans & Google Maps \\
9 & Material & Google Maps \\
10 & Average daily traffic & ANAS data \\
11 & Type of bypassed entity & Google Maps \\
\hline
\end{tabular}

The structure-foundation exposure will be determined based on the average daily traffic, average span length, presence of road alternatives and type of bypassed entity. As an example, the presence of a railway (or a highway) under the bridge results in higher exposure, while the presence of a secondary road or a river will result in medium exposure.

Parameters for the determination of the seismic class of attention are very similar to the parameters needed for the structure-foundation one, with some differences (Table 4). 
Table 4. Parameters influencing seismic risk.

\begin{tabular}{ccc}
\hline $\begin{array}{c}\text { Seismic Risk } \\
\text { Component }\end{array}$ & $\begin{array}{c}\text { Primary } \\
\text { Parameters }\end{array}$ & $\begin{array}{c}\text { Secondary } \\
\text { Parameters }\end{array}$ \\
\hline Hazard & $\begin{array}{c}\text { Expected peak ground acceleration (return } \\
\text { period Tr }=475 \text { y) }\end{array}$ & Soil class \\
\hline Vulnerability & $\begin{array}{c}\text { Level of degradation, static scheme max span } \\
\text { length, material }\end{array}$ & $\begin{array}{c}\text { Design according to seismic } \\
\text { criteria }\end{array}$ \\
\hline Exposure & Average daily traffic and mean span length & $\begin{array}{c}\text { Road alternatives } \\
\text { Type of bypassed entity } \\
\text { Transport of dangerous goods } \\
\text { Strategic function }\end{array}$ \\
\hline
\end{tabular}

The main difference is related to hazard, depending on the expected peak ground acceleration that is taken from the Italian hazard map [49] based on a bridge's geographic coordinates that is combined with the topographic condition. The ground type to be considered for each single bridge structure is not directly available. However, a study was devoted to building a national map of Vs30 values with a spatial resolution of $50 \times 50 \mathrm{~m} \mathrm{[50].} \mathrm{This}$ made it possible to create an application named Seismic Soil Class Italy (SSC-Italy) [51] that is able to provide the ground type based on the geographic coordinates, which has been used in this study to retrieve the ground type for each bridge considered in the following methodology application.

As for vulnerability, the primary parameters are evaluated with respect to structural components having an influence on the seismic behaviour, while a secondary parameter is represented by the earthquake resistant design level. In particular, the bridges designed considering seismic actions have to be distinguished from the more vulnerable bridges designed only relative to gravity loads. To this end, the construction period will be helpful in identifying the adopted design code and related criteria. Finally, the possible strategic function for civil protection purposes must be considered. In the Basilicata region, due to their low number, all primary roads and highways have to be considered strategic in case of emergencies related to natural disasters. In order to obtain seismic hazard, vulnerability and exposure, data reported in Table 3 must be combined according to Figure 4a,b reported in the Guidelines [15].

More specifically, the data collection for the application of the Guidelines' procedure is represented in the flowchart of Figure 8. As can be observed, starting from OpenStreetMap, a first bridge database is obtained by filtering data by region and road name. Afterwards, a completeness check is necessary in order to find missing bridge structures. At this point, the geographical coordinates of each bridge are known and can be used to generate links to bridge location in Google Maps. Hence, the subsequent collection of information can be carried out to obtain the full bridge inventory. Finally, the CoA evaluation can be performed. 


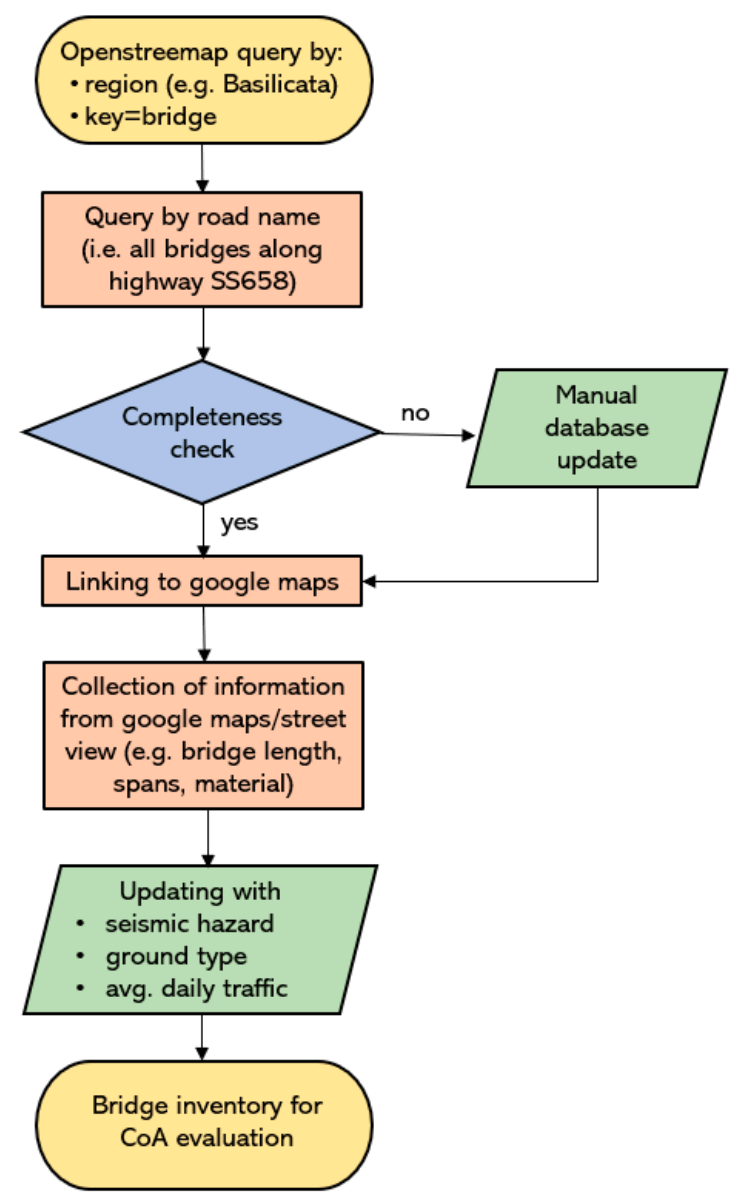

Figure 8. Procedure for bridge inventory.

\subsection{Analysis}

Information on hazard, vulnerability and exposure levels (from Low to High) has to be combined according to Table 4 of the Guidelines in order to obtain the structure-foundation and seismic class of attention result, which is expressed through the same five-level scale.

In order to permit a synthetic and more friendly representation of the results of these combinations, ordinal values can be assigned to hazard, vulnerability and exposure and to the results in terms of class of attention, according to Table 5.

Table 5. Ordinal values assigned to hazard, vulnerability, exposure and class of attention (structurefoundation and seismic).

\begin{tabular}{cc}
\hline $\begin{array}{c}\text { Level of Hazard, Vulnerability, Exposure and Class of } \\
\text { Attention (Structure-Foundation and Seismic) }\end{array}$ & Ordinal Value \\
\hline Low (L) & 1 \\
Medium-Low (M-L) & 2 \\
Medium (M) & 3 \\
Medium-High (M-H) & 4 \\
High (H) & 5 \\
\hline
\end{tabular}

In this manner, the framework for attributing the class of attention for both structurefoundation and seismic risks proposed by the Guidelines can be represented by a 3D histogram, as displayed in Figure 9, which is the graphical translation of the Guidelines' Table 4. 


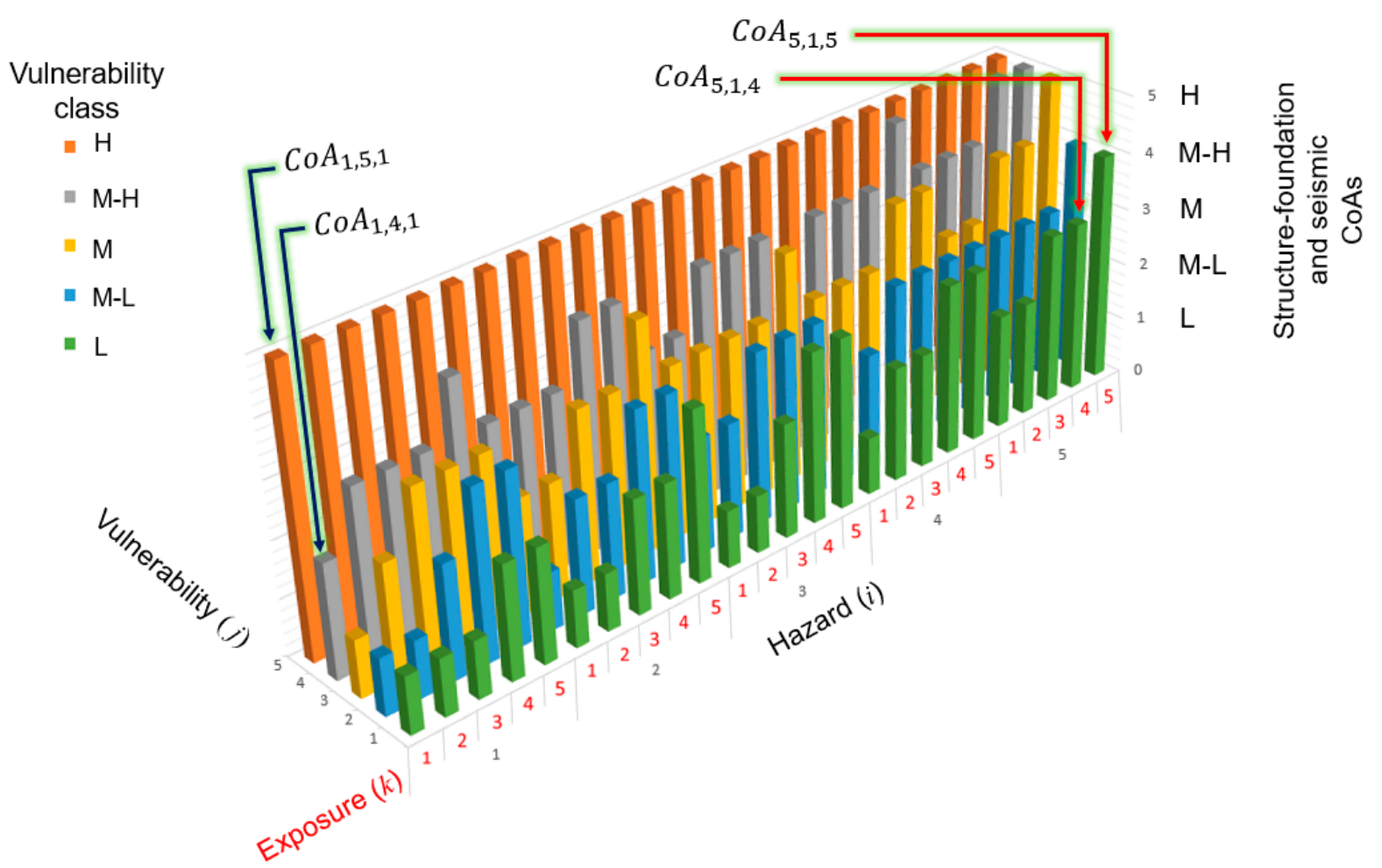

Figure 9. Determination of the structure-foundation and seismic class of attention based on hazard, vulnerability and exposure classes.

It is worth noting that when the vulnerability class is high, structure-foundation and seismic class of attention (CoA) results are always high (orange bars in Figure 9). The rationale of this choice is that vulnerability is essentially influenced by the presence of severe degradation phenomena or defects; thus, rapid and detailed assessments and/or interventions are in any case required.

In order to evaluate the influence of hazard, vulnerability and exposure on the structure-foundation and seismic CoA results, it is helpful to assess the variation of CoA by changing only one of the three parameters at a time while maintaining constant the other parameters. Specifically, the indexes $\mathrm{I}_{\mathrm{H}}, \mathrm{I}_{\mathrm{V}}$ and $\mathrm{I}_{\mathrm{E}}$ are introduced to measure the relative influence of hazard $(\mathrm{H})$, vulnerability $(\mathrm{V})$ and exposure $(\mathrm{E})$, respectively. They are computed by using Equations (1)-(3) (where $i, j$ and $k$ are related to hazard, vulnerability and exposure, respectively, and represent the five possible levels varying in the range 1-5).

$$
\begin{aligned}
I_{H} & =\frac{\sum_{i=2}^{5} \sum_{j=1}^{5} \sum_{k=1}^{5}\left(\operatorname{Co} A_{i, j, k}-\operatorname{Co} A_{i-1, j, k}\right)}{100} \\
I_{V} & =\frac{\sum_{i=1}^{5} \sum_{j=2}^{5} \sum_{k=1}^{5}\left(\operatorname{Co} A_{i, j, k}-\operatorname{Co} A_{i, j-1, k}\right)}{100} \\
I_{E} & =\frac{\sum_{i=1}^{5} \sum_{j=1}^{5} \sum_{k=2}^{5}\left(\operatorname{Co} A_{i, j, k}-\operatorname{Co} A_{i, j, k-1}\right)}{100}
\end{aligned}
$$

As an example, when $i=5$ and $j=1$, that is the maximum hazard and minimum vulnerability; the impact of increasing exposure from $k=4$ to $k=5$, in computing $I_{E}$, is as follows:

$$
\operatorname{Co} A_{i, j, k}-\operatorname{Co}_{i, j, k-1}=\operatorname{Co}_{5,1,5}-\operatorname{Co}_{5,1,4}=1
$$

as can be observed from Figure 9 (red arrows). 
Similarly, an example of computation related to the impact of increasing one vulnerability class to assess $I_{V}$ can be obtained by assuming $i=1$ and $k=1$ and varying $j$ from 4 to 5; this is described as follows:

$$
\operatorname{Co} A_{i, j, k}-\operatorname{Co}_{i, j-1, k}=\operatorname{Co} A_{1,5,1}-\operatorname{Co} A_{1,4,1}=3
$$

(see black arrows in Figure 9).

The denominator in Equations (1)-(3) is equal to 100, as given by the product of the $i, j$ and $k$ summation ranges, which is the following: $5 \times 5 \times 4=100$.

Equations (1)-(3) provide the following results:

- $\quad I_{H}=0.27 ; \max \left(\operatorname{Co}_{i, j, k}-\operatorname{Co}_{i-1, j, k}\right)=1.0$;

- $I_{V}=0.74 ; \max \left(\operatorname{Co}_{i, j, k}-C_{0} A_{i, j-1, k}\right)=3.0$;

- $I_{E}=0.31 ; \max \left(\operatorname{Co}_{i, j, k}-\operatorname{Co}_{i, j, k-1}\right)=1.0$.

The increase in one vulnerability class causes a change of the structure-foundation and seismic CoA that averagely equals to 0.74 . Hazard and exposure possess lower influence, being able to cause $\mathrm{CoA}$ increments that are averagely equal to 0.27 and 0.31 , respectively. Moreover, vulnerability increments are able to cause the maximum CoA increases equal to 3.0. Therefore, according to the Guidelines' approach, vulnerability has a higher impact on the evaluation of the structure-foundation and seismic CoAs.

In order to determine the overall CoA of the bridge at hand, the flooding-landslide CoA needs to be evaluated and combined with structure-foundation and seismic CoAs. Figure 10 shows the approach for combining the four CoAs proposed by the Guidelines (Table 4 in [15]) in order to obtain the overall CoA of a bridge. As can be observed, bridges with a high structure-foundation $\mathrm{CoA}$ always possess a high overall CoA. In fact, the structure-foundation is the highest influencing CoA among the four considered.

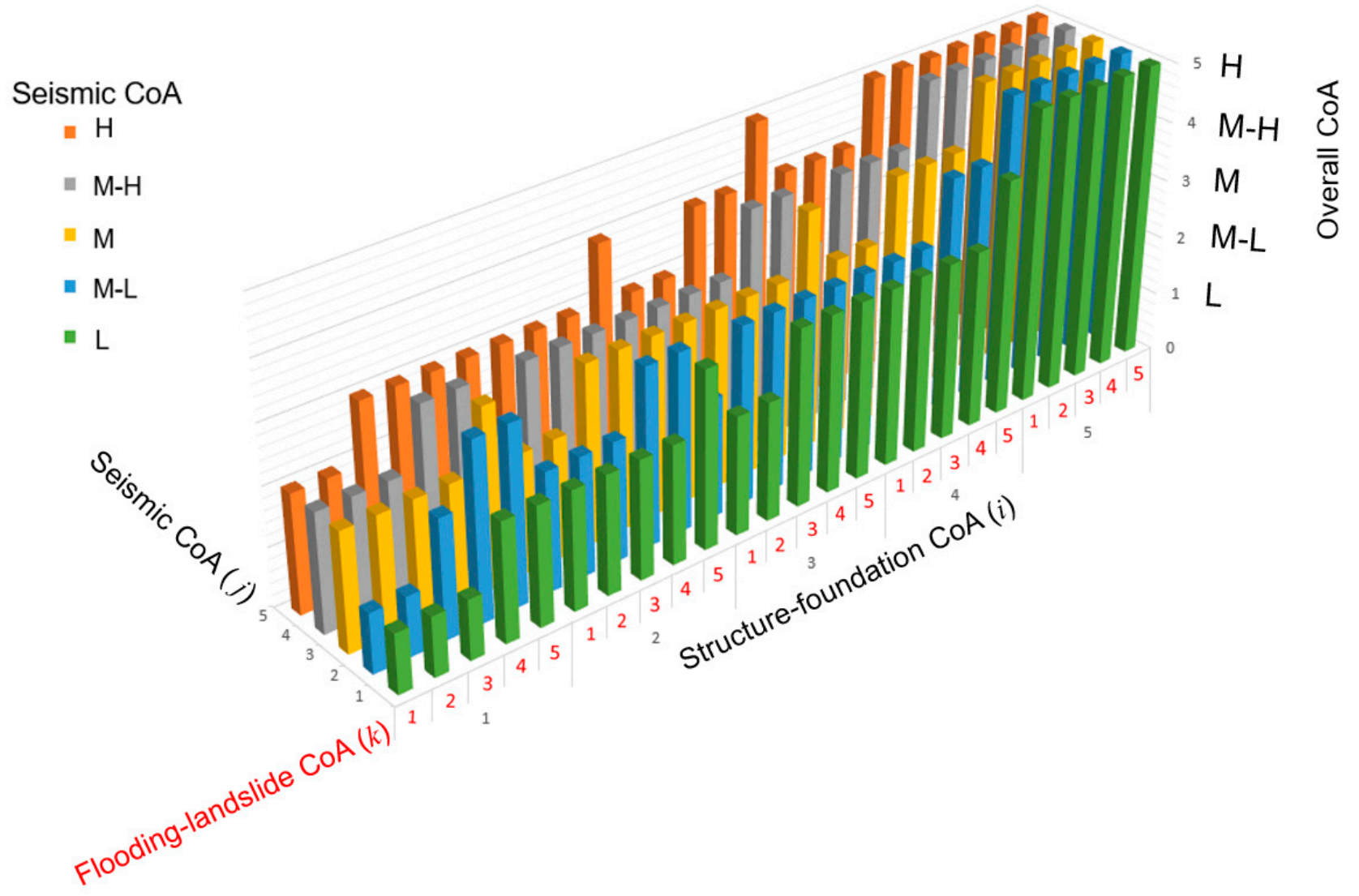

Figure 10. Overall class of attention as a function of structure-foundation, seismic and flooding-landslide CoAs. 
In order to quantitatively evaluate the relative influence factors of structure-foundation $\left(I_{S F}\right)$, seismic $\left(I_{S}\right)$ and flooding-landslide $\left(I_{F L}\right)$ on the CoA results, equations similar to Equations (1)-(3) have been set.

In this case, $i, j$ and $k$ represent the five possible levels (from 1 to 5 ) related to structurefoundation, seismic and flooding-landslide CoA, respectively.

Therefore, Equations (4)-(6) are obtained specifying that, in this case, $C_{0} A_{i, j, k}$ is the overall CoA. As before, the denominator is always equal to 100 .

$$
\begin{gathered}
I_{S F}=\frac{\sum_{i=2}^{5} \sum_{j=1}^{5} \sum_{k=1}^{5}\left(\operatorname{Co} A_{i, j, k}-\operatorname{Co} A_{i-1, j, k}\right)}{100} \\
I_{S}=\frac{\sum_{i=1}^{5} \sum_{j=2}^{5} \sum_{k=1}^{5}\left(\operatorname{Co} A_{i, j, k}-\operatorname{Co} A_{i, j-1, k}\right)}{100} \\
I_{F L}=\frac{\sum_{i=1}^{5} \sum_{j=1}^{5} \sum_{k=2}^{5}\left(\operatorname{Co} A_{i, j, k}-\operatorname{Co} A_{i, j, k-1}\right)}{100}
\end{gathered}
$$

The results from Equations (4)-(6) are as follows.

- $\quad I_{S F}=0.72 ; \max \left(\operatorname{Co}_{i, j, k}-\operatorname{Co}_{i-1, j, k}\right)=2.0$;

- $I_{S}=0.23 ; \max \left(\operatorname{Co} A_{i, j, k}-\operatorname{Co}_{i, j-1, k}\right)=1.0$;

- $I_{F L}=0.22 ; \max \left(\operatorname{Co} A_{i, j, k}-C_{0} A_{i, j, k-1}\right)=1.0$.

The variation of one structure-foundation $\mathrm{CoA}$ is able to averagely induce a variation of the overall CoA higher than those related to the variation of one seismic or floodinglandslide CoA. This means that the structure-foundation CoA has a higher influence on the overall CoA and, thus, has higher impacts on the subsequent measures to be adopted on the bridge at hand.

\subsection{Proposal for Prioritization}

Based on the qualitative approach of the Italian Guidelines, several bridge structures could have the same $\mathrm{CoA}$, making it difficult to establish a rational decision-making process for prioritizing bridges for further assessment or interventions. A prioritization method is necessary to exploit data which has not already been accounted for in the determination of the CoA. In fact, data needed to determine the structure-foundation, seismic, flooding and landslide risks has already provided its contribution to the classification of bridges.

The Guidelines' risk classification can be counted among the worst-conditioned component approaches, where a single severely damaged structural element can induce a high structure-foundation and/or seismic CoA and, therefore, a high overall CoA. On the other hand, the Guidelines also mention the possibility to compute some weighted damage average among all the bridge components to compare the level of degradation of different bridges in a stock for prioritization purposes. However, no details are provided about such a method, which considers only the current level of degradation that in turn is unknown in this specific case, preventing the application of other above-mentioned methods [6]. An effective prioritization method should consider both the current levels of degradation and that expected in the residual lifetime of the bridge, which depends on several parameters related to the intrinsic characteristics of the structure (static scheme, material and span length, etc.) and on external factors related to environmental conditions [52]. Among the latter, the geographic location (for climatic conditions), the elevation on sea level (related to snow probability and use of de-icing salts) and the distance from the sea (related to the presence of chloride aerosols) should at least be accounted for. These environmental parameters are important in the case of prioritization of large bridge stocks located at very different sites, for example, the ANAS bridge stock which includes about 13,000 bridges distributed throughout the Italian peninsula. For a small bridge stock such as the one considered in this study, the previously mentioned environmental conditions do not vary 
significantly and, therefore, a simpler prioritization method can be set by exploiting some of the bridge features not considered in the attribution of the overall CoA.

In fact, the Guidelines do not account for the total length of bridges. Seismic and structure-foundation vulnerability is determined as a function of the maximum span length, deck material and static scheme. The resulting level of vulnerability must be increased by one class only when the total number of spans is higher than three. As an example, this means that all other conditions being equal, bridge $A$ and bridge $B$ having 4 and 20 spans, respectively, will have the same vulnerability class and, therefore, class of attention. On the other hand, it must be considered that the total length can be a useful parameter for measuring the response complexity of a bridge, especially under seismic actions. In fact, bridges with large length are likely to be founded on various ground types, having different local site effects and possible asynchronous seismic excitation [53].

Moreover, disruption due to heavy maintenance works, inspections and material testing to increase the structural knowledge for detailed assessment activities is significantly lower for dual bridges, corresponding to the ones with separate structures for the two different carriageways.

For this kind of bridges, also in the case of a closed carriageway (Figure 11), the open one can be switched to a two-way carriageway. The result is that, even in absence of effective detours, works can be carried out on a carriageway at a time, keeping travel times and discomfort to users sufficiently low.

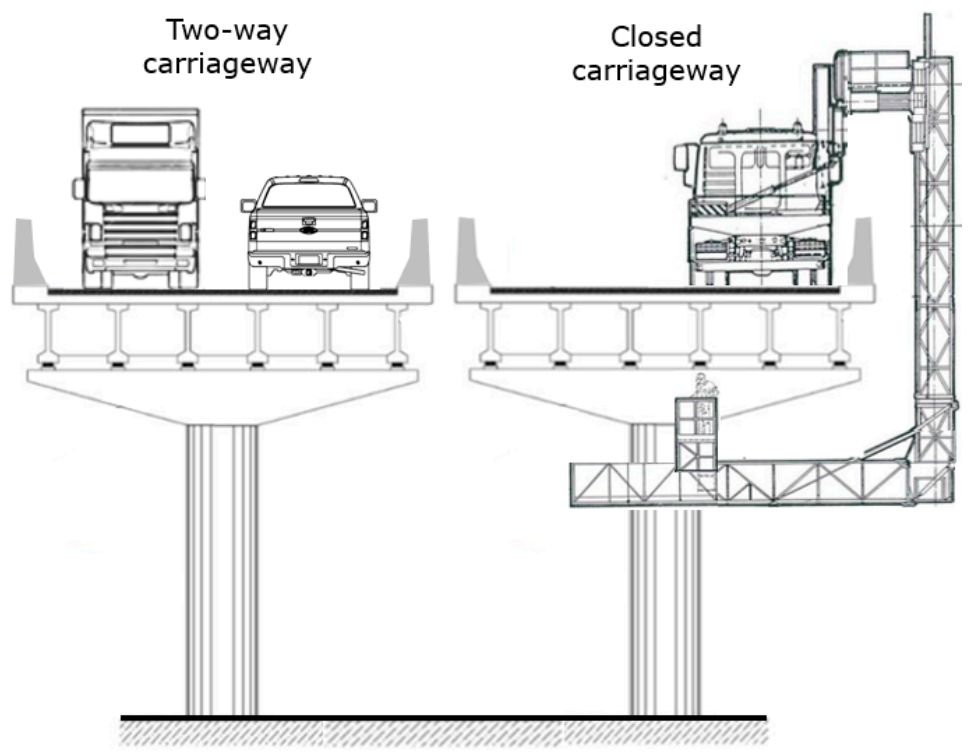

Figure 11. Dual bridge with a closed carriageway.

Therefore, a prioritization method is proposed that acts on bridges organized by groups represented by the classes of attention results (Figure 12). As known, each group includes a number of bridges that cannot be ranked since they all have the same urgency of further assessment or intervention (see Figure 6). In this case, bridges inside each group can be ranked by using the priority index $p_{i}$ defined as follows:

$$
p_{i}=L_{i} \times d_{i}
$$

where $p_{i}$ and $L_{i}$ are the priority index and the total length (in kilometres) of the $i$-th bridge, respectively, with the limitation $L_{i} \leq 1 \mathrm{~km} ; d_{i}$ is the duality coefficient with $d_{i}=0.5$ for dual bridges and $d_{i}=1.0$ for non-dual bridges. 


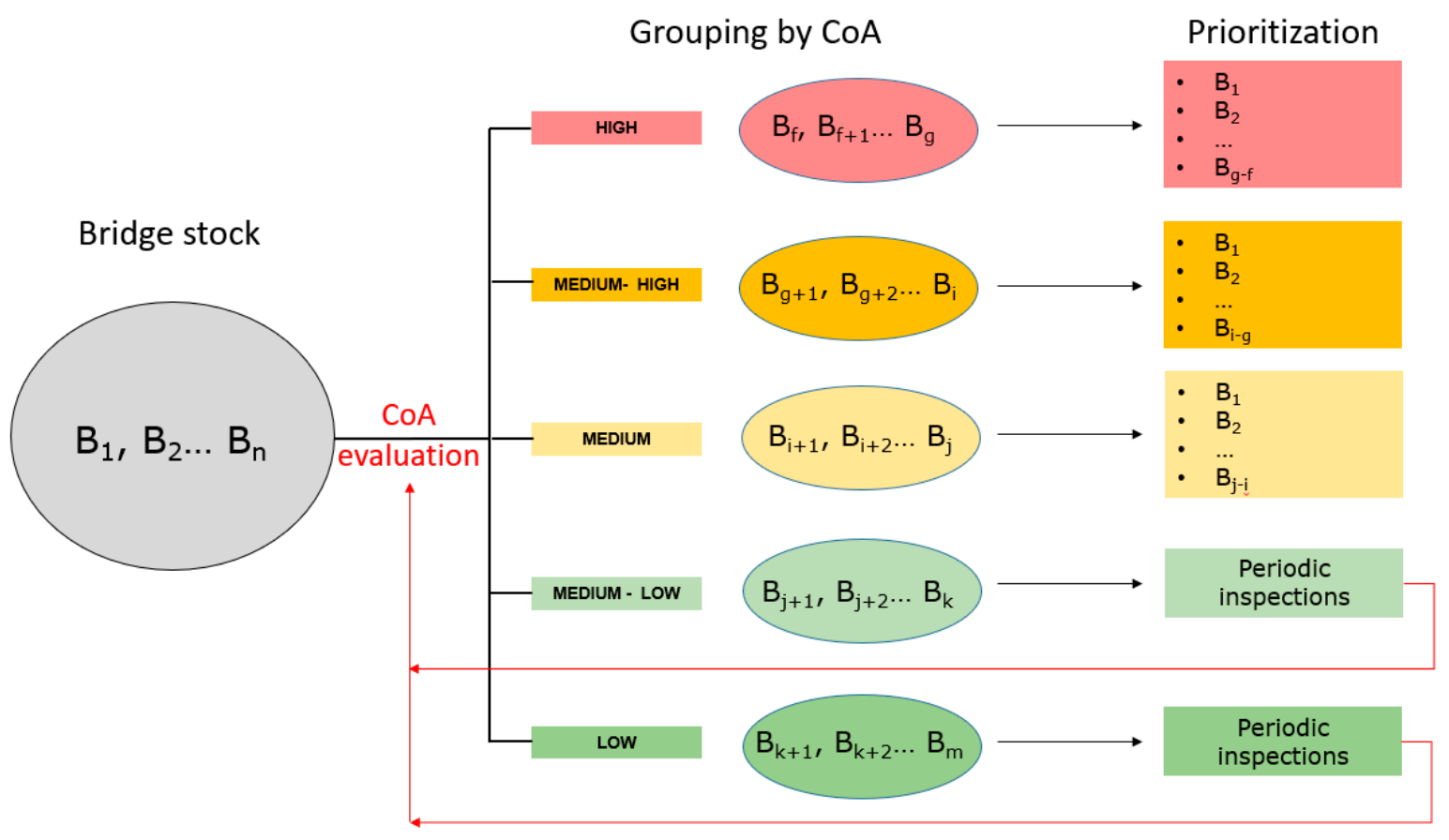

Figure 12. Prioritization of bridges in a stock.

The maximum value of $p_{i}$ is 1.0 since, for bridges longer than $1.0 \mathrm{~km}$, it will be $L_{i}=1.0 \mathrm{~km}$ and $d_{i} \leq 1.0$.

Bridges are then sorted by decreasing $p_{i}$ values, and this does not apply to bridges with medium-low or low class of attention since they may be used without specific measures until the next periodic inspection and the consequent class of attention update (Figure 12).

The priority rankings are valid only inside each class of attention group. This is because, according to Figure 6, different measures must be adopted depending on the class of attention of a bridge (preliminary assessment, detailed assessment, special inspections and permanent monitoring).

However, given the limited financial resources, it reasonable that first expenditures are allocated for bridges with a higher class of attention and then for the other classes.

\section{Results and Discussion}

The application of the semiautomated bridge data collection developed in this study allowed the bridge inventory to be built. While the OpenStreetMaps queries are immediate (Figure 8), the subsequent Google Maps/Street View data retrieval requires manual operations which take a couple of working days for a skilled structural engineer. The required time effort includes the completeness check and is valid for a small size inventory as in this case. Proportionally increased time should be considered for larger stocks. The main weakness of the data collection procedure is related to the difficulty in the assessing the degradation level. This latter difficulty happens because useful Google Street View images are not always available or sufficient for reliably estimating degradation throughout the bridge and its components. For this reason, given the objective of this study (testing the Guidelines procedure through an application), the parameter related to deterioration is assumed.

This does not affect to actual aim of this study that is focused on applying the new Italian BMS to find out possible drawbacks or limitations rather that making a real risk classification. Thus, in order to evaluate the results of the entire procedure in determining the overall CoA, either low (L) or medium-low (M-L) levels of degradation have been essentially assumed. Figures 13-15 show the structure-foundation, seismic and overall CoA (in terms of numerical values), respectively. 


$$
5
$$

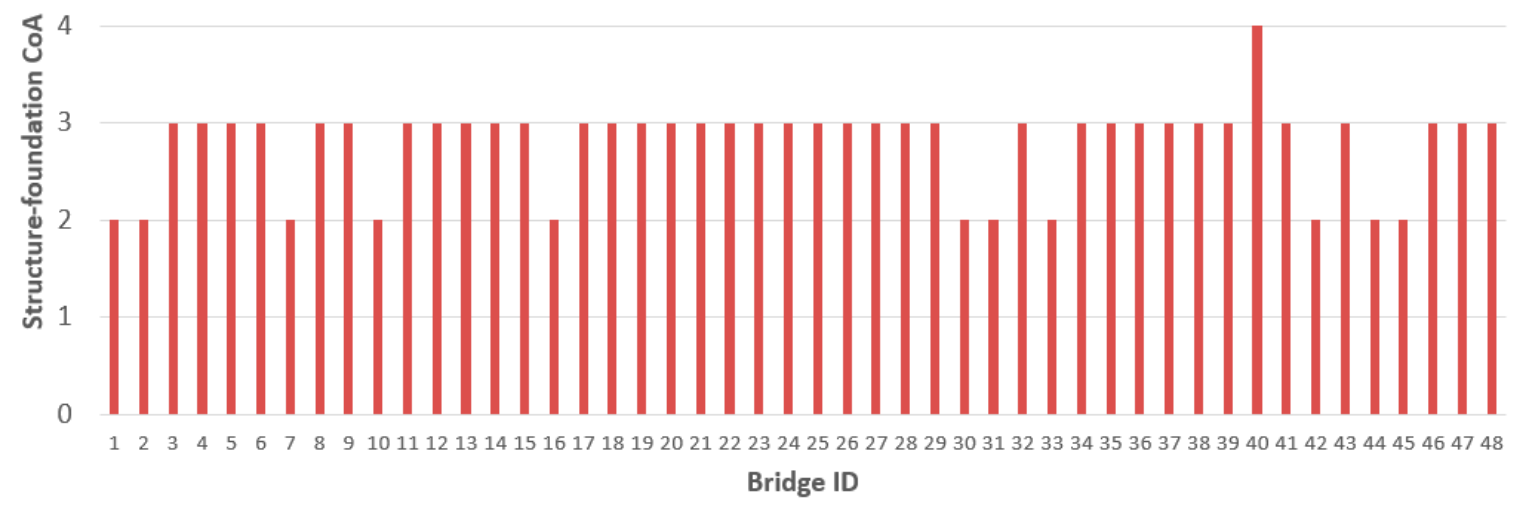

Figure 13. Structure-foundation CoA.

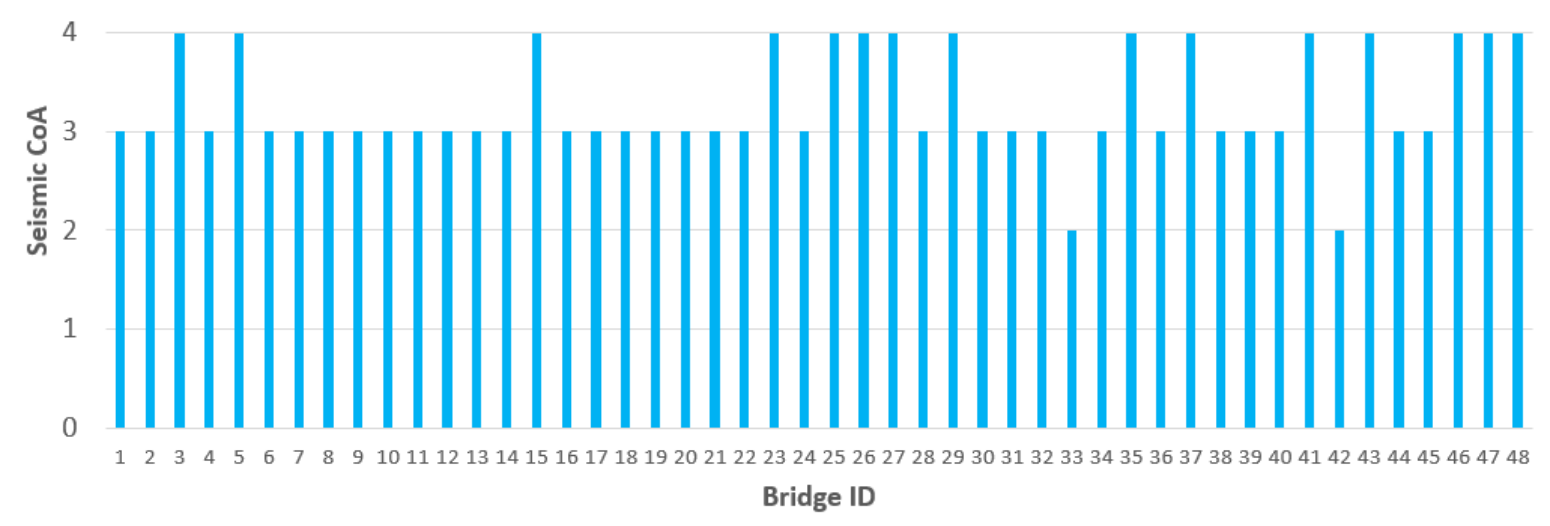

Figure 14. Seismic CoA.

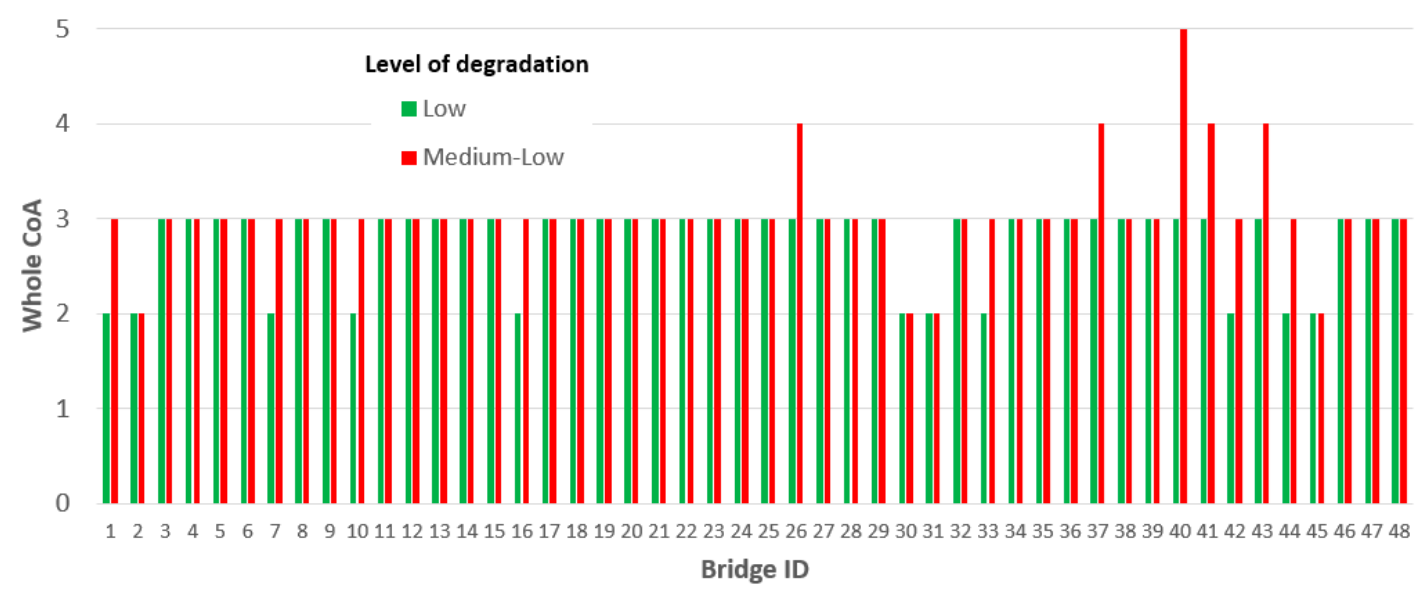

Figure 15. Overall CoA in case of low and medium-low levels of degradation.

As can be observed from Figure 13, the structure-foundation CoA is almost always 3 (Medium), while few bridges have 2 (medium-low) and one bridge (ID = 40) has 4 (medium-high).

Seismic CoA is basically medium (3) with several medium-high values (4) (Figure 14). This is mainly due to the fact that most bridges were designed with respect to gravity loads 
only and to the rather high seismic hazard. In fact, the average expected peak ground acceleration with 475 years return period is equal to $\mathrm{ag}=0.17 \mathrm{~g}$. As a consequence, 37 bridges out of 48 have a medium overall CoA, meaning that they at least need a preliminary assessment and cannot remain in service by only performing periodic inspections. Eleven bridge structures have a medium-low overall CoA, whereas no structure has a low CoA (Figure 15). This suggests that the Guidelines, in their current version, can provide results unbalanced towards high risk levels.

It is worth noting that bridges with low level of degradation are only a few. In fact, expeditious surveys made by the authors on ten bridges (out of 48) and information retrieved by the Google Street View pictures highlight that in several cases, at least a medium-low level of degradation should be assigned.

Assuming a medium-low level of degradation for all the 48 bridge structures, results in terms of overall CoA are reported in Table 6 and Figure 15 along with those related to a low level of degradation.

Table 6. Overall CoA result as a function of the level of degradation.

\begin{tabular}{ccc}
\hline \multirow{2}{*}{ Overall CoA } & \multicolumn{2}{c}{ Level of Degradation } \\
Low & 0 & 0 \\
Low & 11 & 4 \\
Medium-low & 37 & 39 \\
Medium & 0 & 4 \\
Medium-high & 0 & 1 \\
High & 0 & Medium-Low \\
\hline
\end{tabular}

With this latter assumption, only four bridge structures have medium-low CoA. Thirtynine have medium (needing a preliminary evaluation along with special inspections), four possess medium-high (preliminary evaluation, special inspections and permanent monitoring) and one possesses high (permanent monitoring and detailed assessment).

It is worth noting that bridges with medium-high or high overall CoA have a total length of $5.12 \mathrm{~km}$ equal to $38 \%$ of the total bridges' development along SS658 highway.

The 39 bridges needing at least a preliminary evaluation (medium CoA) have a total length of $8.32 \mathrm{~km}$ ( $62 \%$ of the total bridges length). The four bridges with medium-low CoA are small overpasses with negligible length.

As for the prioritization of bridges for further assessment actions (according to the Guidelines provisions based on the class of attention), the application of the proposed procedure yields the ranking shown in Table 7, evaluated in the hypothesis of low degradation level. No bridge in the stock is dual and, therefore, prioritization reflects the total length. It is worth noting that all bridges longer than $1 \mathrm{~km}$ would have the same priority if they belong to the same class of attention. As can be observed from Table 7, also bridges with medium-low overall CoA (equal to 2) have been ranked even though they do not need measures. 
Table 7. Priority ranking for low degradation level.

\begin{tabular}{|c|c|c|c|c|c|c|c|c|}
\hline ID & Rank & $\begin{array}{c}\text { Total } \\
\text { Length }(\mathrm{m})\end{array}$ & $\begin{array}{c}\text { Mean } \\
\text { Span } \\
\text { Length (m) }\end{array}$ & $\begin{array}{c}\text { Static } \\
\text { Scheme } \\
\left.\quad{ }^{*}\right)\end{array}$ & $\begin{array}{c}\text { Material } \\
\qquad(* *)\end{array}$ & $\begin{array}{l}\text { Construction } \\
\text { Period }\end{array}$ & $\begin{array}{c}\text { Overall } \\
\text { CoA }\end{array}$ & Priority-Pi \\
\hline 40 & 1 & 4270 & 36 & SSG & SRC & $>1980$ & 3 & 1 \\
\hline 38 & 2 & 962 & 33 & SSG & PRC & $<1980$ & 3 & 0.962 \\
\hline 17 & 3 & 475 & 34 & SSG & PRC & $<1980$ & 3 & 0.475 \\
\hline 8 & 4 & 469 & 34 & SSG & PRC & $<1980$ & 3 & 0.469 \\
\hline 34 & 5 & 467 & 33 & SSG & PRC & $<1980$ & 3 & 0.467 \\
\hline 26 & 6 & 466 & 33 & SSG & PRC & $<1980$ & 3 & 0.466 \\
\hline 11 & 7 & 412 & 52 & SSG & STEEL & $<1980$ & 3 & 0.412 \\
\hline 9 & 8 & 355 & 44 & SSG & STEEL & $<1980$ & 3 & 0.355 \\
\hline 14 & 9 & 339 & 34 & SSG & PRC & $<1980$ & 3 & 0.339 \\
\hline 19 & 10 & 328 & 30 & SSG & PRC & $<1980$ & 3 & 0.328 \\
\hline 35 & 11 & 300 & 33 & SSG & PRC & $<1980$ & 3 & 0.300 \\
\hline 24 & 12 & 298 & 33 & SSG & PRC & $<1980$ & 3 & 0.298 \\
\hline 6 & 13 & 286 & 36 & SSG & PRC & $<1980$ & 3 & 0.286 \\
\hline 24 & 14 & 271 & 34 & SSG & PRC & $<1980$ & 3 & 0.271 \\
\hline 15 & 15 & 238 & 34 & SSG & PRC & $<1980$ & 3 & 0.238 \\
\hline 22 & 16 & 195 & 33 & SSG & PRC & $<1980$ & 3 & 0.195 \\
\hline 43 & 17 & 188 & 38 & SSG & PRC & $<1980$ & 3 & 0.188 \\
\hline 4 & 18 & 182 & 46 & SSG & STEEL & $<1980$ & 3 & 0.182 \\
\hline 32 & 19 & 170 & 34 & SSG & PRC & $<1980$ & 3 & 0.170 \\
\hline 13 & 20 & 169 & 34 & SSG & PRC & $<1980$ & 3 & 0.169 \\
\hline 46 & 21 & 165 & 33 & SSG & PRC & $<1980$ & 3 & 0.165 \\
\hline 3 & 22 & 135 & 34 & SSG & PRC & $<1980$ & 3 & 0.135 \\
\hline 25 & 23 & 103 & 34 & SSG & PRC & $<1980$ & 3 & 0.103 \\
\hline 41 & 24 & 103 & 34 & SSG & PRC & $<1980$ & 3 & 0.103 \\
\hline 27 & 25 & 100 & 33 & SSG & PRC & $<1980$ & 3 & 0.100 \\
\hline 37 & 26 & 100 & 33 & SSG & PRC & $<1980$ & 3 & 0.100 \\
\hline 47 & 27 & 100 & 33 & SSG & PRC & $<1980$ & 3 & 0.100 \\
\hline 21 & 28 & 99 & 33 & SSG & PRC & $<1980$ & 3 & 0.099 \\
\hline 21 & 29 & 99 & 33 & SSG & PRC & $<1980$ & 3 & 0.099 \\
\hline 5 & 30 & 98 & 33 & SSG & PRC & $<1980$ & 3 & 0.098 \\
\hline 29 & 31 & 98 & 33 & SSG & PRC & $<1980$ & 3 & 0.098 \\
\hline 39 & 32 & 90 & 30 & SSG & PRC & $<1980$ & 3 & 0.090 \\
\hline 18 & 33 & 66 & 33 & SSG & PRC & $<1980$ & 3 & 0.066 \\
\hline 20 & 34 & 65 & 33 & SSG & PRC & $<1980$ & 3 & 0.065 \\
\hline 48 & 35 & 45 & 23 & SSG & PRC & $<1980$ & 3 & 0.045 \\
\hline 28 & 36 & 35 & 35 & SSG & PRC & $<1980$ & 3 & 0.035 \\
\hline 31 & 37 & 4 & 4 & SSS & $\mathrm{RC}$ & $<1980$ & 3 & 0.004 \\
\hline 7 & 38 & 536 & 34 & SSG & PRC & $<1980$ & 2 & 0.536 \\
\hline 45 & 39 & 508 & 34 & SSG & PRC & $<1980$ & 2 & 0.508 \\
\hline 16 & 40 & 35 & 12 & $\mathrm{CS}$ & $\mathrm{RC}$ & $<1980$ & 2 & 0.035 \\
\hline 1 & 41 & 11 & 11 & SSS & $\mathrm{RC}$ & $<1980$ & 2 & 0.011 \\
\hline 44 & 42 & 10 & 10 & SSS & $\mathrm{RC}$ & $<1980$ & 2 & 0.010 \\
\hline 30 & 43 & 8 & 8 & FS & $\mathrm{RC}$ & $<1980$ & 2 & 0.008 \\
\hline 42 & 44 & 7 & 7 & FS & $\mathrm{RC}$ & $<1980$ & 2 & 0.007 \\
\hline 31 & 45 & 6 & 6 & SSS & $\mathrm{RC}$ & $<1980$ & 2 & 0.006 \\
\hline 10 & 46 & 6 & 6 & FS & $\mathrm{RC}$ & $<1980$ & 2 & 0.006 \\
\hline 33 & 47 & 6 & 6 & SSS & $\mathrm{RC}$ & $<1980$ & 2 & 0.006 \\
\hline 2 & 48 & 6 & 6 & FS & $\mathrm{RC}$ & $<1980$ & 2 & 0.006 \\
\hline
\end{tabular}

* SSG: simply supported girders; SSS: simply supported slab; FS: fixed slab; CS: continuous span. ${ }^{* *}$ PRC: prestressed reinforced concrete; SRC: steel-reinforced concrete (mixed); RC: reinforced concrete.

\section{Conclusions}

The paper presents a procedure for risk classification of road bridges based on the approach proposed by Italian Guidelines for bridge assessment, which is applied to a bridge stock located in the Basilicata region (southern Italy). The procedure is presented after the description of some methods for bridge health assessment used worldwide. It is 
worth underlining that the new Italian approach is able to take into account all the internal (bridge vulnerability) and external (flooding and landslide hazards) factors affecting the whole risk.

The study also proposes a semiautomated data collection procedure able to build the bridge inventory by exploiting the open road database OpenStreetMap (which allows automatic bridge detection) plus manual data retrieval by using Google Maps and Street View data (manually) to perform remote surveys, measure bridge dimensions and determine other necessary parameters such as the static scheme, material and so on. Moreover, data on the ground from the seismic point of view can be retrieved by using the SSC-Italy procedure.

Once the bridge inventory was built, the procedure for risk classification proposed by the Guidelines was applied to the 48 bridge structures on the SS 658 highway (Basilicata region, Italy) by assuming data related to degradation levels, which are not available (or not reliable) from a remote survey. The aim of this application is to find possible drawbacks of the Guidelines approach accounting for the fact that they have not been widely applied in Italy since their issuing. Therefore, results cannot be considered a real risk classification of the examined bridges, also considering that some basic information is assumed.

The result of the Guidelines' procedure is expressed in terms of class of attention according to five increasing levels proportional to the global bridge risk (structure-foundation, seismic, flooding and landslide).

Even assuming low degradation levels for all the bridges, most of them (37 out of 48) would be classified in medium $\mathrm{CoA}$, meaning that they should undergo a preliminary assessment and special inspections. It is worth noting that, if preliminary assessment results do not guarantee proper safety levels, detailed assessment activities become mandatory. Moreover, in assuming a slightly heavier level of degradation (medium-low), which is highly probable for many bridges in the stock, five bridge structures would result in medium-high or high CoA. These five bridges have a total length equal to about $38 \%$ of the total bridges' length along the examined road route. This would mean that assessment or intervention measures are necessary on the whole bridge stock in order to hold serviceability and safety, despite the low level of degradation assumed, resulting in the conclusion that the Guidelines' approach is somewhat conservative. However, the major limit of the Guidelines' approach is the qualitative nature of the classification result that groups the bridges at hand into a maximum of five classes of attention, making it difficult to build a priority list. For this reason, a prioritization method based on bridge data not properly exploited in the class of attention attribution procedure is proposed. Data used to prioritize the bridges with the same class of attention are represented by the bridge's total length and the roadway duality. It is worth remembering that the proposed prioritization method applies to relatively small bridge stocks, with rather uniform environmental conditions. The application of this latter method is useful in ranking bridges belonging to same class of attention.

Finally, it is worth underlining that this is a preliminary study where low levels of flooding and landslide risks are assumed. Therefore, more detailed analyses that take these risks (involving other experts) into account are desirable, in addition to considering possible amplifying effects related to climate change. At the same time, it can be considered that the adopted approach for data gathering related to bridge inventory and risk classification can be applied in other countries.

Author Contributions: Conceptualization, G.S., A.M. and V.P.; methodology, G.S., A.M. and V.P.; software, G.S., A.M., V.P., A.D.; validation, G.S., V.P., A.D.; formal analysis, G.S., A.M., V.P., A.D.; investigation, G.S., V.P., A.D.; resources, V.P., A.D.; data curation, G.S., V.P., A.D.; writing—original draft preparation, G.S., A.M., V.P.; writing-review and editing, G.S., A.M., V.P., A.D.; visualization, G.S., A.M., V.P., A.D.; supervision, G.S., A.M.; project administration, G.S., A.M.; funding acquisition, G.S., A.M. All authors have read and agreed to the published version of the manuscript.

Funding: This study received no funding. 
Institutional Review Board Statement: Not applicable.

Informed Consent Statement: Not applicable.

Data Availability Statement: Not applicable.

Acknowledgments: The authors wish to express their gratitude to the four anonymous reviewers for the comments and suggestions provided, which have been very helpful in improving the paper.

Conflicts of Interest: The authors declare no conflict of interest.

\section{References}

1. Pregnolato, M. Bridge safety is not for granted-A novel approach to bridge management. Eng. Struct. 2019, 196, 109193. [CrossRef]

2. Calvi, G.M.; Moratti, M.; O’Reilly, G.J.; Scattarreggia, N.; Monteiro, R.; Malomo, D.; Calvi, P.M.; Pinho, R. Once upon a Time in Italy: The Tale of the Morandi Bridge. Struct. Eng. Int. 2019, 29, 198-217. [CrossRef]

3. Bazzucchi, F.; Restuccia, L.; Ferro, G.A. Considerations over the Italian road bridge infrastructure safety after the Polcevera viaduct collapse: Past errors and future perspectives. Frat. Integrità Strutt. 2018, 46, 400-421. [CrossRef]

4. Santarsiero, G.; Masi, A.; Picciano, V. Durability of Gerber Saddles in RC Bridges: Analyses and Applications (Musmeci Bridge, Italy). Infrastructures 2021, 6, 25. [CrossRef]

5. Pellegrino, C.; Pipinato, A.; Modena, C. A simplified management procedure for bridge network maintenance. Struct. Infrastruct. Eng. 2011, 7, 341-351. [CrossRef]

6. Synthesis of National and International Methodologies Used for Bridge Health Indices; U.S. Department of Transportation, Federal Highway Administration: McLean, VA, USA, 2016.

7. Hadjidemetriou, G.M.; Herrera, M.; Parlikad, A.K. Condition and criticality-based predictive maintenance prioritisation for networks of bridges. Struct. Infrastruct. Eng. 2021. [CrossRef]

8. Dinh, T.H. Maintenance prioritization method for networked bridges. Struct. Infrastruct. Eng. 2009, 5, 381-394. [CrossRef]

9. Sasmal, S.; Ramanjaneyulu, K.; Lakshmanan, N. Priority ranking towards condition assessment of existing reinforced concrete bridges. Struct. Infrastruct. Eng. 2007, 3, 75-89. [CrossRef]

10. Rokneddin, K.; Ghosh, J.; Dueñas-Osorio, L.; Padgett, J.E. Bridge retrofit prioritisation for ageing transportation networks subject to seismic hazards. Struct. Infrastruct. Eng. 2013, 9, 1050-1066. [CrossRef]

11. NTC2018-Ministry of Infrastructure Decree, DM 17 gennaio 2018: Aggiornamento delle Norme tecniche per le costruzioni, Suppl. or. n.30 alla G.U. n.29 del 4/2/2008 (in Italian), 2018.

12. CIRCOLARE 21 Gennaio 2019, n. 7 C.S.LL.PP. Istruzioni per L'applicazione dell' «Aggiornamento Delle “Norme Tecniche per le Costruzioni"» di cui al Decreto Ministeriale 17 Gennaio 2018 (Commentary of NTC2018).

13. EN 1998-2:2005 Eurocode 8: Design of Structures for Earthquake Resistance—Part 2: Bridges; European Committee for Standardization: Brussels, Belgium, 2005.

14. EN 1998-3:2005 Eurocode 8: Design of Structures for Earthquake Resistance-Part 3: Assessment and Retrofitting of Buildings; European Committee for Standardization: Brussels, Belgium, 2005.

15. Guidelines on Risk Classification and Management, Safety Assessment and Monitoring of Existing Bridges; Ministry of Infrastructure, CSLP: Rome, Italy, 2020.

16. Ministry of Infrastructure Decree no. 578 del 17/12/2020. Enforcement of the Guidelines on risk classification and management safety assessment and monitoring of existing bridges (in Italian: Adozione delle linee guida per la gestione del rischio dei ponti esistenti e per la definizione di requisiti ed indicazioni relativi al sistema di monitoraggio dinamico).

17. Mooney, P.; Minghini, M. A Review of OpenStreetMap Data. In Mapping and the Citizen Sensor; Foody, G., See, L., Fritz, S., Mooney, P., Olteanu-Raimond, A.-M., Fonte, C.C., Antoniou, V., Eds.; Ubiquity Press: London, UK, 2017; pp. 37-59. [CrossRef]

18. Karlaftis, M.; Kepaptsoglou, K.; Lambropoulos, S. A web-supported national bridge inventory management tool. Public Work. Manag. Policy 2005, 9, 248-258. [CrossRef]

19. Hyeonsoo, K.; Changwan, K. Deep-Learning-Based Classification of Point Clouds for Bridge Inspection. Remote Sens. 2020, 12, 3757. [CrossRef]

20. Liu, H.; Zhang, Y. Bridge condition rating data modeling using deep learning algorithm. Struct. Infrastruct. Eng. 2020, 16, 1447-1460. [CrossRef]

21. Marra, A.; Fabbrocino, G. Open-access web mapping as a virtual survey tool for cultural heritage: An application to the Armenian religious architecture documented by Paolo Cuneo. Disegnarecon 2020, 13. [CrossRef]

22. Wang, N.; Zhao, X.; Wang, L.; Zou, Z. Novel System for Rapid Investigation and Damage Detection in Cultural Heritage Conservation Based on Deep Learning. J. Infrastruct. Syst. 2019, 25. [CrossRef]

23. Maniat, M.; Camp, C.V.; Kashani, A.R. Deep learning-based visual crack detection using Google Street View images. Neural Comput. Applic. 2021. [CrossRef]

24. Marra, A.; Fabbrocino, G. Crowd-Based Tools for Indirect Condition Assessment and Conservation of Cultural Heritage. EuroMed 2020, 12642. [CrossRef] 
25. Spencer, B.F., Jr.; Hoskere, V.; Narazaki, Y. Advances in Computer Vision-Based Civil Infrastructure Inspection and Monitoring. Engineering 2019, 5, 199-222. [CrossRef]

26. Narazaki, Y.; Hoskere, V.; Hoang, T.A.; Spencer, B.F., Jr. Vision-based Automated Bridge Component Recognition Integrated with High-level Scene Understanding. In Proceedings of the 13th International Workshop on Advanced Smart Materials and Smart Structures Technology, Tokyo, Japan, 22-23 July 2017.

27. Mangalathu, S.; Soleimani, F.; Jeon, J.-S. Bridge classes for regional seismic risk assessment: Improving HAZUS models. Eng. Struct. 2017, 148, 755-766. [CrossRef]

28. Whelan, M.J.; Cavalline, T.L.; Alar, A.; Lane, K. Guidelines for Prioritization of Bridge Replacement, Rehabilitation, and Preservation Projects; University of North Carolina at Charlotte: Charlotte, NC, USA, 2019.

29. Valenzuela, S.; De Solminihac, H.; Echaveguren, T. Proposal of an Integrated Index for Prioritization of Bridge Maintenance. J. Bridge Eng. 2009. [CrossRef]

30. Shepard, R.W.; Johnson, M.B. California Bridge Health Index: A Diagnostic Tool to Maximize Bridge Longevity, Investment. TR News 2001, 215.

31. Mohamed Mansour, D.M.; Moustafa, I.M.; Khalil, A.H.; Mahdi, H.A. An assessment model for identifying maintenance priorities strategy for bridges. Ain Shams Eng. J. 2019, 10, 695-704. [CrossRef]

32. Rashidi, M.; Samali, B.; Sharafi, P. A new model for bridge management: Part A: Condition assessment and priority ranking of bridges. Aust. J. Civ. Eng. 2016, 14, 35-45. [CrossRef]

33. Abu Dabous, S.; Hamad, K.; Al-Ruzouq, R. Defect-Based Urgency Index for Bridge Maintenance Ranking and Prioritization. Int. J. Civ. Environ. Eng. 2016, 10, 605-609.

34. Yokoyama, K.; Sato, H.; Ogihara, K.; Toriumi, R. Development of a Bridge Management System in Japan. In Proceedings of the Third International Conference on Bridge Management, London, UK, 14-17 April 1996; pp. 580-594.

35. Omar, T.; Nehdi, M.L. Condition Assessment of Reinforced Concrete Bridges: Current Practice and Research Challenges. Infrastructures 2018, 3, 36. [CrossRef]

36. Ercolessi, S.; Fabbrocino, G.; Rainieri, C. Indirect Measurements of Bridge Vibrations as an Experimental Tool Supporting Periodic Inspections. Infrastructures 2021, 6, 39. [CrossRef]

37. Abdallah, A.M.; Atadero, R.A.; Ozbek, M.E. A Comprehensive Uncertainty-Based Framework for Inspection Planning of Highway Bridges. Infrastructures 2021, 6, 27. [CrossRef]

38. Savini, F.; Rainieri, C.; Fabbrocino, G.; Trizio, I. Applications of Stratigraphic Analysis to Enhance the Inspection and Structural Characterization of Historic Bridges. Infrastructures 2021, 6, 7. [CrossRef]

39. Ilbeigi, M.; Pawar, B. A Probabilistic Model for Optimal Bridge Inspection Interval. Infrastructures 2020, 5, 47. [CrossRef]

40. Rainieri, C.; Notarangelo, M.A.; Fabbrocino, G. Experiences of Dynamic Identification and Monitoring of Bridges in Serviceability Conditions and after Hazardous Events. Infrastructures 2020, 5, 86. [CrossRef]

41. Bukhsh, Z.A.; Oslakovic, I.S.; Klanker, G.; Hoj, N.P.; Imam, B.; Xenidis, Y. Multi-criteria decision making: AHP method applied for network bridge prioritization. In Proceedings of the Joint COST TU1402-COST TU1406-IABSE WC1 Workshop, Zagreb, Croatia, 2-3 March 2017; pp. 1-9.

42. Saaty, T.L. The Analytic Hierarchy Process Mcgraw Hill, New York. Agric. Econ. Rev. 1980, 70.

43. Saaty, T.L. Decision Making for Leaders: The Analytic Hierarchy Process for Decision in a Complex World; RWS Publications: Pittsburgh, PA, USA, 1999.

44. ANAS. Available online: https://www.stradeanas.it/it/le-strade/la-rete-anas (accessed on 15 May 2021).

45. AINOP. Available online: https:/ / ainop.mit.gov.it/portale\#/ (accessed on 15 July 2021).

46. ISTAT-National Institute of Statistics. Conti Economici Territoriali (Territorial Economic Accounts). 2020. Available online: https:/ / www.istat.it/it/archivio/237813 (accessed on 15 March 2021).

47. ISTAT-National Institute of Statistics. Main Geostatistical Data and Level of Urbanization of Italy (in Italian: Principali Dimensioni Geostatistiche e Grado di Urbanizzazione del Paese). Available online: https://www.istat.it/it/archivio/137001 (accessed on 15 March 2021).

48. ANAS. Dati di Traffico Medio Giornaliero Annuale (Average Daily Traffic Data per Year). Available online: https://www. stradeanas.it/it/le-strade/osservatorio-del-traffico/dati-traffico-medio-giornaliero-annuale (accessed on 15 March 2021).

49. Stucchi, M.; Meletti, C.; Montaldo, V.; Crowley, H.; Calvi, G.M.; Boschi, E. Seismic hazard assessment (2003-2009) for the Italian building code. Bull Seism. Soc. Am. 2011, 101, 1885-1911. [CrossRef]

50. Mori, F.; Mendicelli, A.; Moscatelli, M.; Romagnoli, G.; Peronace, E.; Naso, G. A new Vs30 map for Italy based on the seismic microzonation dataset. Eng. Geol. 2020, 275, 105745. [CrossRef]

51. Forte, G.; Chioccarelli, E.; De Falco, M.; Cito, P.; Santo, A.; Iervolino, I. Seismic soil classification of Italy based on surface geology and shear-wave velocity measurements. Soil Dyn. Earthq. Eng. 2019, 122, 79-93. [CrossRef]

52. Mannella, P. Inspections and Maintenance for Bridge Durability: Management Criteria, Code Framework and Intervention Methods. 28 March 2019. Available online: https:/ / seewebstorage.it/alig/ANAS-Mannella.pdf (accessed on 15 June 2021).

53. Sextos, A.G.; Kappos, A.J. Evaluation of seismic response of bridges under asynchronous excitation and comparisons with Eurocode 8-2 provisions. Bull Earthq. Eng. 2009, 7, 519. [CrossRef] 